\title{
STABILITY CRITERIA FOR VOLTERRA EQUATIONS
}

\author{
BY
}

\section{T. A. BURTON AND W. E. MAHFOUD}

ABSTRACT. We consider a system of integro-differential equations of the form

$$
x^{\prime}=A(t) x+\int_{0}^{t} C(t . s) x(s) d s
$$

with $A$ and $C$ being $n \times n$ matrices. Various types of stability are defined and results are obtained showing when one type of stability is equivalent to another type. We also construct a number of Lyapunov functionals from which we obtain necessary and sufficient conditions for stability of (1.1). Finally, we prove several results concerning qualitative behavior of solutions of (1.1).

Introduction. We consider the integro-differential equation

$$
x^{\prime}=A(t) x+\int_{0}^{t} C(t, s) x(s) d s
$$

in which $A$ is an $n \times n$ matrix continuous for $0 \leqslant t<\infty$ and $C$ is an $n \times n$ matrix continuous for $0 \leqslant s \leqslant t<\infty$.

The purpose of this paper is to investigate relations between stability properties of solutions of (1.1) under various assumptions on $A, C$, and the dimension $n$. We will also give necessary and sufficient conditions for stability of solutions of (1.1) and necessary and sufficient conditions for stability and boundedness of solutions of some perturbed forms of (1.1).

It is known that, for the ordinary differential equation

$$
x^{\prime}=D(t) x
$$

with $D(t)$ an $n \times n$ matrix of functions continuous and $T$-periodic on $[0, \infty)$, stability, uniform stability, and boundedness of solutions are equivalent concepts. Furthermore, asymptotic stability, uniform asymptotic stability, $L^{1}[0, \infty)$ stability, and boundedness of all solutions of

$$
x^{\prime}=D(t) x+F(t)
$$

for every continuous and bounded $F$ \} are all equivalent to the property that all solutions of (1.2) tend to zero as $t \rightarrow \infty$. (The $T$-periodic assumption is stronger than necessary as may be seen in Hale [5, pp. 152-153].)

We are interested here in equivalent statements for (1.1) and their implications for a forced version of (1.1).

Stability definitions given below are from Driver [4] and Miller [9]. Existence, uniqueness, and continuation results are found in Driver [4].

Received by the editors August 23, 1982.

1980 Mathematics Subject Classification. Primary 45D05, 45J05; Secondary 34K20.

(C) 1983 American Mathematical Society 0002-9947/82/0000-1330/\$09.00 
A solution of $(1.1)$ with continuous initial function $\varphi:\left[0, t_{0}\right] \rightarrow R^{n}$ will be denoted by $x\left(t, t_{0}, \varphi\right), x(t, \varphi)$, or just $x(t)$ if no confusion should arise.

If $D$ is a matrix or a vector, $|D|$ means the sum of the absolute values of the elements.

If the function is written without its argument, then that argument is always understood to be $t$.

Definition 1. The zero solution of (1.1) is stable if for every $\varepsilon>0$ and any $t_{0} \geqslant 0$ there exists a $\delta>0$ such that $\left[|\varphi(t)|<\delta\right.$ on $\left[0, t_{0}\right]$ and $\left.t \geqslant t_{0}\right]$ imply $\left|x\left(t, t_{0}, \varphi\right)\right|<\varepsilon$.

Definition 2. The zero solution of (1.1) is uniformly stable if it is stable and the above $\delta$ is independent of $t_{0}$.

Definition 3. The zero solution of (1.1) is asymptotically stable if it is stable and if for each $t_{0} \geqslant 0$ there is an $\eta>0$ such that $|\varphi(t)|<\eta$ on $\left[0, t_{0}\right]$ implies $x\left(t, t_{0}, \varphi\right) \rightarrow 0$ as $t \rightarrow \infty$.

Definition 4. The zero solution of (1.1) is uniformly asymptotically stable if it is uniformly stable, the above $\eta$ is independent of $t_{0}$, and for every $\varepsilon>0$ there is a $T(\varepsilon)>0$ such that $\left[|\varphi(t)|<\eta\right.$ on $\left[0, t_{0}\right]$ and $\left.t \geqslant t_{0}+T\right]$ imply $\left|x\left(t, t_{0}, \varphi\right)\right|<\varepsilon$.

Definition 5. The zero solution of $(1.1)$ is unstable if it is not stable.

DEFINITION 6. The zero solution of (1.1) is completely unstable if there exists an $\varepsilon>0$ and a $t_{0} \geqslant 0$ such that for any $\delta>0, \delta<\varepsilon$ if $\varphi:\left[0, t_{0}\right] \rightarrow R^{n}$ is continuous, if $\varphi(t) \not 0$, and if $|\varphi(t)|<\delta$, then there is a $t_{1}>t_{0}$ with $\left|x\left(t_{1}, t_{0}, \varphi\right)\right| \geqslant \varepsilon$.

Definition 7. An $n \times n$ matrix is said to be stable if all of its characteristic roots have negative real parts.

2. Stability. The following four papers will have significant bearing on our investigation.

I. In [9] R. K. Miller considered (1.1) with $A$ constant and $C=C(t-s)$ and showed that if $Z(t)$ is the $n \times n$ matrix satisfying

$$
Z^{\prime}(t)=A Z(t)+\int_{0}^{t} C(t-s) Z(s) d s, \quad Z(0)=I,
$$

and if

$$
\int_{0}^{\infty}|C(t)| d t<\infty
$$

then $Z(t) \in L^{1}[0, \infty)$ if and only if the zero solution of (1.1) is uniformly asymptotically stable. He did not require that $A$ be a stable matrix.

II. In [2] F. Brauer asked that (1.1) be a scalar equation with $A$ constant and $C(t, s)=C(t-s)$. He assumed that

$$
\begin{aligned}
& \int_{0}^{\infty}|C(v)| d v<\infty, \\
& \int_{0}^{\infty} t|C(t)| d t<\infty,
\end{aligned}
$$

and proved the following result.

(a) If $C(t)>0$ and $A+\int_{0}^{\infty} C(t) d t<0$, then the zero solution of (1.1) is uniformly asymptotically stable. 
(b) If $C(t)<0, A+\int_{0}^{\infty} C(t) d t<0$, and $\int_{0}^{\infty} t|C(t)| d t$ is sufficiently small, then the zero solution of (1.1) is uniformly asymptotically stable.

(c) If $A+\int_{0}^{\infty} C(t) d t \geqslant 0$, the zero solution of (1.1) is not uniformly asymptotically stable.

III. In [3] Burton showed that if $A$ is constant and

$$
C=C(t-s) \text { with } A \text { stable and } \int_{t}^{\infty}|C(u-t)| d u \text { small, }
$$

then

$$
\int_{0}^{\infty}|Z(t)| d t<\infty
$$

Thus, (2.5) implies uniform asymptotic stability by I above.

IV. In [8] Levin considered a nonlinear scalar version of

$$
x^{\prime}(t)=-\int_{0}^{t} a(t-s) x(s) d s
$$

and asked that

$$
(-1)^{k} a^{(k)}(t) \geqslant 0 \text { for } 0 \leqslant t<\infty
$$

and $k=0,1,2,3$ with $a(t) \not a(0)$. He concluded that $x^{(j)}(t) \rightarrow 0$ as $t \rightarrow \infty$ for $j=0,1,2$.

Our first result is quite special as it concerns a scalar convolution equation with strong sign conditions, but it is thought provoking.

We consider the scalar equation

$$
x^{\prime}=A x+\int_{0}^{t} C(t-s) x(s) d s
$$

in which $A$ is constant and $C(t)$ is continuous for $0 \leqslant t<\infty$.

Theorem 1. Suppose $A<0, C(t)>0$, and $A+\int_{0}^{\infty} C(t) d t \neq 0$. The following statements are equivalent:

(i) All solutions of (2.7) tend to zero.

(ii) $A+\int_{0}^{\infty} C(t) d t<0$.

(iii) Each solution of (2.7) is in $L^{1}[0, \infty)$.

(iv) The zero solution of (2.7) is uniformly asymptotically stable.

(v) The zero solution of (2.7) is asymptotically stable.

Proof. We show that $(n)$ implies $(n+1)$. Of course, (v) implies (i).

Suppose (i) holds, but $A+\int_{0}^{\infty} C(t) d t>0$. Choose $t_{0}$ so large that $A+\int_{0}^{t_{0}} C(s) d s$ $>0$ and let $\varphi(t)=2$ on $\left[0, t_{0}\right]$. Then we claim that $x(t, \varphi)>1$ on $\left[t_{0}, \infty\right)$. If not, then there is a first $t_{1}$ with $x\left(t_{1}\right)=1$ and, therefore, $x^{\prime}\left(t_{1}\right) \leqslant 0$. But

$$
\begin{aligned}
x^{\prime}\left(t_{1}\right) & =A x\left(t_{1}\right)+\int_{0}^{t_{1}} C\left(t_{1}-s\right) x(s) d s=A+\int_{0}^{t_{1}} C(s) x\left(t_{1}-s\right) d s \\
& \geqslant A+\int_{0}^{t_{1}} C(s) d s>A+\int_{0}^{t_{0}} C(s) d s>0,
\end{aligned}
$$

a contradiction. Thus, (i) implies (ii). 
Let (ii) hold and consider the Lyapunov functional

$$
V(t, x(\cdot))=|x|+\int_{0}^{t} \int_{t}^{\infty} C(u-s) d u|x(s)| d s .
$$

If $x(t)$ is a solution of (2.7), then for $x \neq 0$ we have

$$
\begin{aligned}
V_{(2.7)}^{\prime}(t, x(\cdot)) \leqslant & A|x|+\int_{0}^{t} C(t-s)|x(s)| d s \\
& +\int_{t}^{\infty} C(u-t) d u|x|-\int_{0}^{t} C(t-s)|x(s)| d s \\
= & {\left[A+\int_{t}^{\infty} C(u-t) d u\right]|x|=\left[A+\int_{0}^{\infty} C(u) d u\right]|x|=-\alpha|x|, }
\end{aligned}
$$

some $\alpha>0$. Thus,

$$
0 \leqslant V(t, x(\cdot)) \leqslant V\left(t_{0}, \varphi(\cdot)\right)-\alpha \int_{t_{0}}^{t}|x(s)| d s,
$$

and so $\int_{t_{0}}^{\infty}|x(s)| d s<\infty$, as required. Thus, (ii) implies (iii).

Now Miller's result (I) shows that (iii) implies (iv), while (iv) certainly implies (v). The proof is complete.

REMARK 1. The analog of the condition with (1.3) may also be inserted in the list of equivalences in Theorem 1. Details of this sort appear in Theorem 7.

REMARK 2. The list of equivalences in Theorem 1 have some very practical applications. If $Z(t)$ satisfes (2.1), then the variation of parameters formula for

$$
x^{\prime}=A x+\int_{0}^{t} C(t-s) x(s) d s+F(t)
$$

with $F:[0, \infty) \rightarrow R^{n}$ being continuous, is

$$
x\left(t, 0, x_{0}\right)=Z(t) x_{0}+\int_{0}^{t} Z(t-s) F(s) d s .
$$

For example, under the conditions of Theorem 1, if $A+\int_{0}^{\infty} C(t) d t<0$, then $Z(t)$ is in $L^{1}[0, \infty), Z(t)$ is bounded, and $Z(t)$ tends to zero as $t \rightarrow \infty$. Thus, from (2.9) we see that:

(a) If $F$ is bounded, then $x\left(t, 0, x_{0}\right)$ is bounded.

(b) If $F$ is in $L^{1}[0, \infty)$, then $x\left(t, 0, x_{0}\right)$ tends to zero as $t \rightarrow \infty$ (as we have the convolution of an $L^{1}$ function $(F)$ with a function tending to zero $\left.(Z(t))\right)$.

(c) If $F(t) \rightarrow 0$ as $t \rightarrow \infty$, then $x\left(t, 0, x_{0}\right) \rightarrow 0$ as $t \rightarrow \infty$ (as we have the convolution of an $L^{1}$ function $(Z(t))$ with a function tending to zero $\left.(F(t))\right)$.

REMARK 3. Theorem 1 shows that Brauer's condition (2.4) was not needed. In fact, by slightly modifying the Lyapunov functional used in the proof of Theorem 1, we can also show that the sign condition on $C(t)$ may be relaxed as it is stated in the following theorem.

TheOREM 2. If $A+\int_{0}^{\infty}|C(t)| d t<0$, then the zero solution of (2.7) is uniformly asymptotically stable.

Proof. Let

$$
V(t, x(\cdot))=|x|+\int_{0}^{t} \int_{t}^{\infty}|C(u-s)| d u|x(s)| d s .
$$


If $x(t)=x\left(t, t_{0}, \varphi\right)$ is a solution of (2.7) then

$$
\begin{aligned}
V_{(2.7)}^{\prime}(t, x(\cdot)) \leqslant & A|x|+\int_{0}^{t}|C(t-s)||x(s)| d s \\
& +\int_{t}^{\infty}|C(u-t)| d u|x|-\int_{0}^{t}|C(t-s)||x(s)| d s \\
\leqslant & {\left[A+\int_{0}^{\infty}|C(u)| d u\right]|x|=-\alpha|x|, \quad \alpha>0 . }
\end{aligned}
$$

Thus, $x(t)$ is in $L^{1}[0, \infty)$ and so the proof is complete.

REMARK 4. One might now question the severity of the condition

$$
A+\int_{0}^{\infty}|C(t)| d t<0
$$

and ask whether or not a more relaxed condition, such as

$$
A+\left|\int_{0}^{\infty} C(t) d t\right|<0
$$

would guarantee the uniform asymptotic stability of the zero solution of (2.7).

The following example will show that the zero solution of (2.7) may be completely unstable under condition (2.10) even if we ask $C(t)$ to be in $L^{1}[0, \infty)$ and bounded.

EXAMPLE 1. Consider the scalar equation

$$
x^{\prime}=-x+\int_{0}^{t} C(t-s) x(s) d s,
$$

where

$$
\begin{aligned}
C(t) & =b \sin t & & \text { if } 0 \leqslant t \leqslant 2 \pi, \\
& =0 & & \text { if } t \geqslant 2 \pi,
\end{aligned}
$$

for some $b>0$.

By writing (2.11) in the form

$$
x^{\prime}=-x+\int_{0}^{t} C(s) x(t-s) d s,
$$

we have

$$
\begin{aligned}
x^{\prime} & =-x+b \int_{0}^{t}(\sin s) x(t-s) d s \quad \text { if } 0 \leqslant t \leqslant 2 \pi, \\
& =-x+b \int_{0}^{2 \pi}(\sin s) x(t-s) d s \quad \text { if } t \geqslant 2 \pi
\end{aligned}
$$

or

$$
\begin{aligned}
x^{\prime} & =-x+b \int_{0}^{t}[\sin (t-s)] x(s) d s \quad \text { if } 0 \leqslant t \leqslant 2 \pi, \\
& =-x+b \int_{t-2 \pi}^{t}[\sin (t-s)] x(s) d s \quad \text { if } t \geqslant 2 \pi .
\end{aligned}
$$

Let $t_{0}=2 \pi$ and $\varphi(t)=\lambda e^{t-2 \pi}$ on $\left[0, t_{0}\right], \lambda$ constant.

We will show that for some choice of $b, x(t)=\lambda e^{t-t_{0}}$ is a solution of (2.11) with $x(t)=\varphi(t)$ on $\left[0, t_{0}\right]$. 
Since

$$
\int_{t-2 \pi}^{t} e^{s} \sin (t-s) d s=(1 / 2) e^{t}\left(1-e^{-2 \pi}\right)
$$

then for $b=\left[4 e^{2 \pi}\right] /\left(e^{2 \pi}-1\right), x(t)=\lambda e^{t-t_{0}}$ is a solution of $(2.11)$ with $x(t)=\varphi(t)$ on $\left[0, t_{0}\right]$.

Observe that $C(t)$ is bounded, in $L^{1}[0, \infty)$, and (2.10) is satisfied.

REMARK 5. The above example is a counterexample to a statement in Theorem 3 of Jordan's paper [7] where condition (2.10) is stated as a sufficient condition for the zero solution of (2.7) to be uniformly asymptotically stable.

Throughout this paper we will continue the discussion of stability of solutions of (2.7) where a condition such as (2.10) holds, namely $A+\int_{0}^{\infty} C(t) d t<0$.

We now consider a more general scalar equation and give necessary and sufficient conditions for stability of the zero solution.

Let

$$
x^{\prime}=A(t) x+\int_{0}^{t} C(t, s) x(s) d s
$$

in which $A:[0, \infty) \rightarrow R$ is continuous and $C$ is continuous for $0 \leqslant s \leqslant t<\infty$. We assume that $\int_{t}^{\infty}|C(u, t)| d u$ is defined for $t \geqslant 0$.

THEOREM 3. Suppose there is a constant $\alpha>0$ such that

$$
\int_{0}^{t}|C(t, s)| d s+\int_{t}^{\infty}|C(u, t)| d u-2|A(t)| \leqslant-\alpha .
$$

Then (2.12) is stable if and only if $A(t)<0$.

Proof. Suppose $A(t)<0$ and consider the functional

$$
V(t, x(\cdot))=x^{2}+\int_{0}^{t} \int_{t}^{\infty}|C(u, s)| d u x^{2}(s) d s .
$$

The derivative of $V(t, x(\cdot))$ along a solution $x(t)$ of (2.12) satisfies

$$
\begin{aligned}
V_{(2.12)}^{\prime}(t, x(\cdot)) \leqslant & 2 A x^{2}+2 \int_{0}^{t}|C(t, s)||x(s)||x| d s \\
& +\int_{t}^{\infty}|C(u, t)| d u x^{2}-\int_{0}^{t}|C(t, s)| x^{2}(s) d s \\
\leqslant & 2 A x^{2}+\int_{0}^{t}|C(t, s)|\left(x^{2}(s)+x^{2}\right) d s \\
& +\int_{t}^{\infty}|C(u, t)| d u x^{2}-\int_{0}^{t}|C(t, s)| x^{2}(s) d s \\
= & {\left[2 A+\int_{0}^{t}|C(t, s)| d s+\int_{t}^{\infty}|C(u, t)| d u\right] x^{2} \leqslant-\alpha x^{2} . }
\end{aligned}
$$

As $V$ is positive definite and $V^{\prime} \leqslant 0$ it follows that $x=0$ is stable.

Suppose that $A(t)>0$ and consider the functional

$$
W(t, x(\cdot))=x^{2}-\int_{0}^{t} \int_{t}^{\infty}|C(u, s)| d u x^{2}(s) d s
$$


so that

$$
\begin{aligned}
W_{(2.12)}^{\prime}(t, x(\cdot)) \geqslant & 2 A x^{2}-2 \int_{0}^{t}|C(t, s)||x(s)||x| d s \\
& -\int_{t}^{\infty}|C(u, t)| d u x^{2}+\int_{0}^{t}|C(t, s)| x^{2}(s) d s \\
\geqslant & 2 A x^{2}-\int_{0}^{t}|C(t, s)|\left(x^{2}(s)+x^{2}\right) d s \\
& -\int_{t}^{\infty}|C(u, t)| d u x^{2}+\int_{0}^{t}|C(t, s)| x^{2}(s) d s \\
= & {\left[2 A-\left(\int_{0}^{t}|C(t, s)| d s+\int_{t}^{\infty}|C(u, t)| d u\right)\right] x^{2} \geqslant \alpha x^{2} . }
\end{aligned}
$$

Now, given any $t_{0} \geqslant 0$ and any $\delta>0$, we can find a continuous function $\varphi$ : $\left[0, t_{0}\right] \rightarrow R$ with $|\varphi(t)|<\delta$ and $W\left(t_{0}, \varphi(\cdot)\right)>0$ so that if $x(t)=x\left(t, t_{0}, \varphi\right)$ is a solution of (2.12), then we have

$$
\begin{aligned}
x^{2}(t) & \geqslant W(t, x(\cdot)) \geqslant W\left(t_{0}, \varphi(\cdot)\right)+\alpha \int_{t_{0}}^{t} x^{2}(s) d s \\
& \geqslant W\left(t_{0}, \varphi(\cdot)\right)+\alpha \int_{t_{0}}^{t} W\left(t_{0}, \varphi(\cdot)\right) d s \\
& =W\left(t_{0}, \varphi(\cdot)\right)+\alpha W\left(t_{0}, \varphi(\cdot)\right)\left(t-t_{0}\right) .
\end{aligned}
$$

As $t \rightarrow \infty,|x(t)| \rightarrow \infty$. This completes the proof.

COROLlaRY 1. If (2.13) holds and $A(t)<0$ and bounded, then the zero solution of (2.12) is asymptotically stable.

Proof. We showed in the proof of Theorem 3 that $V_{(2.12)}^{\prime}(t, x(\cdot)) \leqslant-\alpha x^{2}$. This implies that $x^{2}(t)$ is in $L^{1}[0, \infty)$ and $x^{2}(t)$ is bounded. It follows from (2.13) and (2.12) that $x^{\prime}(t)$ is bounded. Thus, $x(t) \rightarrow 0$ as $t \rightarrow \infty$. The proof is now complete.

COROLlary 2. If (2.13) holds and $A(t)>0$, then the zero solution of (2.12) is completely unstable. Furthermore, for any $t_{0} \geqslant 0$ and any $\delta>0$ there is a continuous function $\varphi:\left[0, t_{0}\right] \rightarrow R$ and a solution $x\left(t, t_{0}, \varphi\right)$ with $|\varphi(t)|<\delta$ and

$$
\left|x\left(t, t_{0}, \varphi\right)\right| \geqslant\left[c_{1}+c_{2}\left(t-t_{0}\right)\right]^{1 / 2}
$$

where $c_{1}$ and $c_{2}$ are positive constants depending on $t_{0}$ and $\varphi$.

Proof. This is an immediate consequence of (2.14).

REMARK 6. Notice that condition (2.13) would not hold if $A(t)$ is allowed to vanish at some point $t \geqslant 0$. Therefore, Theorem 3 cannot apply unless $A(t) \neq 0$ for all $t \geqslant 0$. Our next result will consider equation (2.12) where $A(t)$ may vanish at any $t \geqslant 0$. Equations such as (2.12) where $A(t) \equiv 0$ have been considered by Levin [8].

We select a continuous function $G(t, s)$ with

$$
\partial G(t, s) / \partial t=C(t, s)
$$

and let

$$
Q(t)=A(t)-G(t, t)
$$


so that (2.12) may be written as

$$
x^{\prime}=Q(t) x+(d / d t) \int_{0}^{t} G(t, s) x(s) d s .
$$

THEOREM 4. Suppose (2.15) holds and there are constants $Q_{1}, Q_{2}, J$ and $R$ with $R<2$ such that

(i) $0<Q_{1} \leqslant|Q(t)| \leqslant Q_{2}$,

(ii) $\int_{0}^{t}|G(t, s)| d s \leqslant J<1$, and

(iii) $\int_{0}^{t}|G(t, s)| d s+\int_{t}^{x}|G(u, t)| d u \leqslant R Q_{1} / Q_{2}$

for $0 \leqslant t<\infty$. Furthermore, suppose there is a continuous function $h:[0, \infty) \rightarrow[0, \infty)$ with $|G(t, s)| \leqslant h(t-s)$ and $h(u) \rightarrow 0$ as $u \rightarrow \infty$. Then the zero solution is stable if and only if $Q(t)<0$.

Proof. Suppose $Q(t)<0$ and consider the functional

$$
V(t, x(\cdot))=\left(x-\int_{0}^{t} G(t, s) x(s) d s\right)^{2}+Q_{2} \int_{0}^{t} \int_{t}^{\infty}|G(u, s)| d u x^{2}(s) d s .
$$

The derivative of $V$ along a solution $x(t)$ of (2.16) satisfies

$$
\begin{aligned}
V_{(2.16)}^{\prime}(t, x(\cdot))= & 2\left(x-\int_{0}^{t} G(t, s) x(s) d s\right) Q(t) x \\
& +Q_{2} \int_{t}^{\infty}|G(u, t)| d u x^{2}-Q_{2} \int_{0}^{t}|G(t, s)| x^{2}(s) d s \\
\leqslant & 2 Q x^{2}+Q_{2} \int_{0}^{t}|G(t, s)|\left(x^{2}(s)+x^{2}\right) d s \\
& +Q_{2} \int_{t}^{\infty}|G(u, t)| d u x^{2}-Q_{2} \int_{0}^{t}|G(t, s)| x^{2}(s) d s \\
= & {\left[2 Q+Q_{2}\left(\int_{0}^{t}|G(t, s)| d s+\int_{t}^{\infty}|G(u, t)| d u\right)\right] x^{2} } \\
\leqslant & {\left[2 Q+R Q_{1}\right] x^{2} \leqslant\left[-2 Q_{1}+R Q_{1}\right] x^{2}=-\beta x^{2}, \quad \beta>0 . }
\end{aligned}
$$

Let $\varepsilon>0$ and $t_{0} \geqslant 0$ be given. We propose to find $\delta>0$ so that if $|\varphi(t)|<\delta$ on $\left[0, t_{0}\right]$, then $\mid x\left(t, t_{0}, \varphi\right)<\varepsilon$ for all $t \geqslant t_{0}$.

Since $V_{(2.16)}^{\prime}(t, x(\cdot)) \leqslant 0$ for $t \geqslant t_{0}$, then

$$
\begin{aligned}
V(t, x(\cdot)) & \leqslant V\left(t_{0}, \varphi(\cdot)\right) \\
& =\left|\varphi\left(t_{0}\right)-\int_{0}^{t_{0}} G\left(t_{0}, s\right) \varphi(s) d s\right|^{2}+Q_{2} \int_{0}^{t_{0}} \int_{t_{0}}^{\infty}|G(u, s)| d u \varphi^{2}(s) d s \\
& \leqslant \delta^{2}\left[1+\int_{0}^{t_{0}}\left|G\left(t_{0}, s\right)\right| d s\right]^{2}+Q_{2} \delta^{2} \int_{0}^{t_{0}} \int_{t_{0}}^{\infty}|G(u, s)| d u d s \leqslant \delta^{2} N^{2}
\end{aligned}
$$

where

$$
N^{2}=\left(1+R Q_{1} / Q_{2}\right)^{2}+Q_{2} \int_{0}^{t_{0}} \int_{t_{0}}^{\infty}|G(u, s)| d u d s
$$


Now,

$$
V(t, x(\cdot)) \geqslant\left(x(t)-\int_{0}^{t} G(t, s) x(s) d s\right)^{2} \geqslant\left(|x(t)|-\left|\int_{0}^{t} G(t, s) x(s) d s\right|\right)^{2} .
$$

Thus

$$
|x(t)|-\left|\int_{0}^{t} G(t, s) x(s) d s\right| \leqslant \delta N
$$

or

$$
|x(t)| \leqslant \delta N+\int_{0}^{t}|G(t, s)||x(s)| d s .
$$

Now, so long as $|x(t)|<\varepsilon$, we have

$$
|x(t)|<\delta N+\varepsilon \int_{0}^{t}|G(t, s)| d s \leqslant \delta N+J \varepsilon<\varepsilon,
$$

for all $t \geqslant t_{0}$ provided $\delta<\varepsilon(1-J) / N$. Since (2.12) and (2.16) are the same equation, then the zero solution of $(2.12)$ is stable.

Suppose now that $Q(t)>0$ and consider the functional

$$
W(t, x(\cdot))=\left(x-\int_{0}^{t} G(t, s) x(s) d s\right)^{2}-Q_{2} \int_{0}^{t} \int_{t}^{\infty}|G(u, s)| d u x^{2}(s) d s .
$$

Then

$$
\begin{aligned}
W_{(2.16)}^{\prime}(t, x(\cdot))= & 2\left(x-\int_{0}^{t} G(t, s) x(s) d s\right) Q(t) x \\
& -Q_{2} \int_{t}^{\infty}|G(u, t)| d u x^{2}+Q_{2} \int_{0}^{t}|G(t, s)| x^{2}(s) d s \\
\geqslant & 2 Q x^{2}-Q_{2} \int_{0}^{t}|G(t, s)|\left(x^{2}(s)+x^{2}\right) d s \\
& -Q_{2} \int_{t}^{\infty}|G(u, t)| d u x^{2}+Q_{2} \int_{0}^{t}|G(t, s)| x^{2}(s) d s \\
= & {\left[2 Q-Q_{2}\left(\int_{0}^{t}|G(t, s)| d s+\int_{t}^{\infty}|G(u, t)| d u\right)\right] x^{2} } \\
\geqslant & {\left[2 Q_{1}-R Q_{1}\right] x^{2}=\gamma x^{2} }
\end{aligned}
$$

where $\gamma=2 Q_{1}-R Q_{1}$.

Now, given any $t_{0} \geqslant 0$ and any $\delta>0$, we can find a continuous function $\varphi$ : $\left[0, t_{0}\right] \rightarrow R$ with $|\varphi(t)|<\delta$ and $W\left(t_{0}, \varphi(\cdot)\right)>0$ so that if $x(t)=x\left(t, t_{0}, \varphi\right)$ is a solution of (2.12), then we have

$$
\left(x(t)-\int_{0}^{t} G(t, s) x(s) d s\right)^{2} \geqslant W(t, x(\cdot)) \geqslant W\left(t_{0}, \varphi(\cdot)\right)+\gamma \int_{t_{0}}^{t} x^{2}(s) d s .
$$

We will show that $x(t)$ is unbounded. If $x(t)$ is not unbounded, then as $\int_{0}^{t}|G(t, s)| d s$ is bounded, we have $\int_{0}^{t} G(t, s) x(s) d s$ bounded and hence $x^{2}(t)$ is in $L^{1}[0, \infty)$. 
Using the Schwarz inequality, we have

$$
\begin{aligned}
\left(\int_{0}^{t}|G(t, s) \| x(s)| d s\right)^{2} & =\left(\int_{0}^{t}|G(t, s)|^{1 / 2}|G(t, s)|^{1 / 2}|x(s)| d s\right)^{2} \\
& \leqslant \int_{0}^{t}|G(t, s)| d s \int_{0}^{t}|G(t, s)| x^{2}(s) d s \\
& \leqslant \int_{0}^{t}|G(t, s)| d s \int_{0}^{t} h(t-s) x^{2}(s) d s
\end{aligned}
$$

The last integral is the convolution of an $L^{1}$ function with a function tending to zero. Thus the integral tends to zero as $t \rightarrow \infty$ and hence

$$
\int_{0}^{t} G(t, s) x(s) d s \rightarrow 0 \quad \text { as } t \rightarrow \infty .
$$

Since

$$
\left|x-\int_{0}^{t} G(t, s) x(s) d s\right| \geqslant\left[W\left(t_{0}, \varphi(\cdot)\right)\right]^{1 / 2}
$$

then for sufficiently large $T,|x(t)| \geqslant \alpha$ for some $\alpha>0$ and all $t \geqslant T$. This contradicts $x^{2}(t)$ being in $L^{1}[0, \infty)$. Thus, $x(t)$ is unbounded and the zero solution of (2.12) is unstable. This completes the proof.

Let us now apply Theorems 3 and 4 to equation (2.7) so that we may easily evaluate them.

As $C(t, s)=C(t-s)$ and $A(t)=A=$ constant, we assume that $\left|\int_{0}^{\infty} C(v) d v\right|<$ $\infty$ and define $G(t)$ by $G(t)=-\int_{t}^{\infty} C(v) d v$ so that

$$
Q=A-G(0)=A+\int_{0}^{\infty} C(v) d v
$$

Thus, Theorems 3 and 4 take the following forms.

Theorem 3'. Suppose that $\int_{0}^{\infty}|C(v)| d v<|A|$. Then the zero solution of (2.7) is stable if and only if $A<0$.

TheOrem $4^{\prime}$. Suppose that $A+\int_{0}^{\infty} C(v) d v \neq 0$ and

$$
\int_{0}^{\infty}\left|\int_{t}^{\infty} C(v) d v\right| d t<1 .
$$

Then the zero solution of (2.7) is stable if and only if $A+\int_{0}^{\infty} C(v) d v<0$.

Proof. As $Q \neq 0$, we take $Q_{1}=Q_{2}=|Q|$. Now

$$
\int_{0}^{t}|G(t-s)| d s=\int_{0}^{t}|G(v)| d v \leqslant \int_{0}^{\infty}|G(t)| d t=\int_{0}^{\infty}\left|\int_{t}^{\infty} C(v) d v\right| d t<1
$$

and

$$
\int_{t}^{\infty}|G(u-t)| d u=\int_{0}^{\infty}|G(t)| d t<1
$$

Thus, all conditions of Theorem 4 are satisfied. This completes the proof.

Theorem 4' may now be extended as follows. 
THEOREM 5. Suppose that

$$
\int_{0}^{\infty}\left|\int_{t}^{\infty} C(v) d v\right| d t<1
$$

Then the zero solution of (2.7) is stable if and only if $A+\int_{0}^{\infty} C(v) d v \leqslant 0$.

Proof. We need only prove stability when $Q=A+\int_{0}^{\infty} C(v) d v=0$. In this case, (2.7) reduces to $x^{\prime}(t)=(d / d t) \int_{0}^{t} G(t-s) x(s) d s$.

Let $\varepsilon>0$ and $t_{0} \geqslant 0$ be given. We propose to find $\delta>0$ so that if $\varphi:\left[0, t_{0}\right] \rightarrow R$ is continuous with $|\varphi(t)|<\delta$ then $\left|x\left(t, t_{0}, \varphi\right)\right|<\varepsilon$ for $t \geqslant t_{0}$.

By integrating the equation from $t_{0}$ to $t$ we obtain

$$
x(t)=\varphi\left(t_{0}\right)+\int_{0}^{t} G(t-s) x(s) d s-\int_{0}^{t_{0}} G\left(t_{0}-s\right) \varphi(s) d s
$$

and

$$
\begin{aligned}
|x(t)| & \leqslant \delta+\int_{0}^{t}|G(t-s)||x(s)| d s+\delta \int_{0}^{t_{0}}\left|G\left(t_{0}-s\right)\right| d s \\
& \leqslant 2 \delta+\int_{0}^{t}|G(t-s)||x(s)| d s .
\end{aligned}
$$

So long as $|x(t)|<\varepsilon$ we have

$$
|x(t)| \leqslant 2 \delta+\varepsilon \int_{0}^{t}|G(t-s)| d s \leqslant 2 \delta+\varepsilon \int_{0}^{\infty}|G(v)| d v=2 \delta+\varepsilon P,
$$

where $P=\int_{0}^{\infty}|G(v)| d v$. Thus, $|x(t)| \leqslant 2 \delta+\varepsilon P<\varepsilon$ provided that $\delta<\varepsilon(1-P) / 2$. For this choice of $\delta,|x(t)|<\varepsilon$ for all $t \geqslant t_{0}$ and hence $x=0$ is stable. This completes the proof.

Combining Theorems 1 and $4^{\prime}$, we obtain

Theorem 6. If $A<0, C(t)>0, A+\int_{0}^{\infty} C(v) d v \neq 0$ and $\int_{0}^{\infty}\left|\int_{t}^{\infty} C(v) d v\right| d t<1$, then the zero solution of (2.7) is uniformly asymptotically stable if and only if it is stable.

Obviously, Theorem 5 provides a simple and practical way of investigating stability of the zero solution of (2.7). What one would hope to see now is that condition (2.17) is not severe. To this end we present the following example.

EXAmPLE 2. Consider the equation

$$
x^{\prime}=(1-\varepsilon) x+(2 \varepsilon-1) \int_{0}^{t} e^{(2 \varepsilon-1)(t-s)} x(s) d s .
$$

For any $\varepsilon>0$ with $0<\varepsilon<\frac{1}{2}$, the zero solution is unstable, while $A+\int_{0}^{\infty} C(t) d t=$ $-\varepsilon<0$ and $\int_{0}^{\infty}\left|\int_{t}^{\infty} C(v) d v\right| d t=1 /(1-2 \varepsilon)>1$. We may choose $\varepsilon$ as close to zero as we please.

Proof. We may differentiate the equation to obtain

$$
x^{\prime \prime}-\varepsilon x^{\prime}+\varepsilon(1-2 \varepsilon) x=0,
$$

all of whose characteristic roots have positive real parts for $0<\varepsilon<\frac{1}{2}$. Thus, the integrodifferential equation is unstable.

This example shows that assumption (2.17) is very mild and hence Theorem 5 is indeed a sharp one. Furthermore, if (2.17) holds, then for $A<0$ and $C(t)>0$, the 
negation of the inequality in Theorem $3^{\prime}$, that is $\int_{0}^{\infty}|C(t)| d t>|A|$, would imply, by Theorem 5 , that the zero solution is unstable. Consequently, Theorem $3^{\prime}$ is also a sharp one.

In the next section we obtain a modified version of Theorem 5 which also yields a simple and practical criterion for uniform asymptotic stability of the zero solution of (2.7).

3. System. We consider the system

$$
x^{\prime}=A x+\int_{0}^{t} C(t-s) x(s) d s
$$

in which $A$ and $C$ are $n \times n$ matrices, $n \geqslant 1, C(t)$ is continuous for $0 \leqslant t<\infty$, and $A$ is constant.

Let $Z(t)$ be the $n \times n$ matrix satisfying

$$
Z^{\prime}(t)=A Z(t)+\int_{0}^{t} C(t-s) Z(s) d s, \quad Z(0)=I .
$$

THEOREM 7. Suppose there is a constant $M>0$ such that for $0 \leqslant t_{0}<\infty$ and $0 \leqslant t<\infty$ we have

$$
\int_{0}^{t} \int_{0}^{t_{0}}|C(u+v)| d u d v \leqslant M .
$$

Then the following statements are equivalent.

(i) $Z(t) \rightarrow 0$ as $t \rightarrow \infty$.

(ii) All solutions $x\left(t, t_{0}, \varphi\right)$ of (3.1) tend to zero as $t \rightarrow \infty$.

(iii) The zero solution of (3.1) is uniformly asymptotically stable.

(iv) $Z(t)$ is in $L^{1}[0, \infty)$ and $Z(t)$ is bounded.

(v) Every solution $x\left(t, 0, x_{0}\right)$ of

$$
x^{\prime}=A x+\int_{0}^{t} C(t-s) x(s) d s+F(t)
$$

on $[0, \infty)$ is bounded for every bounded and continuous $F:[0, \infty) \rightarrow R^{n}$.

(vi) The zero solution of (3.1) is asymptotically stable.

Furthermore, the following are also equivalent.

(vii) $Z(t)$ is bounded.

(viii) All solutions $x\left(t, t_{0}, \varphi\right)$ of (3.1) are bounded.

(ix) The zero solution of (3.1) is uniformly stable.

(x) The zero solution of (3.1) is stable.

Proof. Let (i) hold. Then a solution $x\left(t, t_{0}, \varphi\right)$ of (3.1) may be considered as a solution of

$$
x^{\prime}=A x+\int_{0}^{t_{0}} C(t-s) \varphi(s) d s+\int_{t_{0}}^{t} C(t-s) x(s) d s
$$

for $t \geqslant t_{0}$ with the second term on the right being treated as a forcing term. If we translate the equation by $y(t)=x\left(t+t_{0}\right)$, we obtain

$$
y^{\prime}(t)=A y(t)+\int_{0}^{t} C(t-s) y(s) d s+\int_{0}^{t_{0}} C\left(t+t_{0}-s\right) \varphi(s) d s .
$$


We may now apply the variation of parameters formula (2.9) and write

$$
y(t)=Z(t) \varphi\left(t_{0}\right)+\int_{0}^{t} Z(t-u) \int_{0}^{t_{0}} C\left(u+t_{0}-s\right) \varphi(s) d s d u .
$$

The substitution $s=t_{0}-v$ yields

$$
y(t)=Z(t) \varphi\left(t_{0}\right)+\int_{0}^{t} Z(t-u) \int_{0}^{t_{0}} C(u+v) \varphi\left(t_{0}-v\right) d v d u .
$$

As $|\varphi(t)| \leqslant K, K>0$, on $\left[0, t_{0}\right]$, we have

$$
|y(t)| \leqslant K|Z(t)|+K \int_{0}^{t}|Z(t-u)| \int_{0}^{t_{0}}|C(u+v)| d v d u .
$$

The last term is the convolution of an $L^{1}[0, \infty)$ function $\left(\int_{0}^{t_{0}}|C(u+v)| d v\right)$ with a function tending to zero $(Z(t))$ and so it tends to zero. Thus, (i) implies (ii).

Suppose that (ii) holds. Then, in particular, all solutions of the form $x\left(t, 0, x_{0}\right)$ tend to zero, implying that $Z(t) \rightarrow 0$ as $t \rightarrow \infty$. Now $\int_{0}^{t} \int_{0}^{t_{0}}|C(u+v)| d u d v \leqslant M$ uniformly in $t_{0}$. Thus the integral $\int_{0}^{t}|Z(t-u)| \int_{0}^{t_{0}}|C(u+v)| d v d u$ tends to zero uniformly in $t_{0}$ and hence $x\left(t, t_{0}, \varphi\right)$ tends to zero uniformly in $t_{0}$ for bounded $\varphi$. Thus, (ii) implies (iii).

Let (iii) hold. Then Miller's result (I) implies that $Z(t)$ is $L^{1}[0, \infty)$. Also, the uniform asymptotic stability implies $Z(t)$ bounded. Hence, (iv) holds.

Suppose (iv) is satisfied. Then solutions $x\left(t, 0, x_{0}\right)$ of $(3.4)$ on $[0, \infty)$ are expressed as

$$
x(t)=Z(t) x(0)+\int_{0}^{t} Z(t-s) F(s) d s
$$

As $Z(t)$ is $L^{1}[0, \infty)$ and bounded and as $F$ is bounded, then $x(t)$ is bounded. Hence, (v) holds.

Suppose (v) is satisfied. Then the argument in the proof of Perron's theorem (cf. Hale [5, p. 152]) yields $Z(t)$ being $L^{1}[0, \infty)$. This, in turn, implies uniform asymptotic stability. Of course, uniform asymptotic stability implies asymptotic stability and so (v) implies (vi).

Certainly, (vi) implies (i). This completes the proof of the first set of equivalences.

Let (vii) hold. The variation of parameters formula implies that $x(t, \varphi)$ is bounded. Thus, (viii) holds.

Suppose (viii) is satisfied. Then $|Z(t)| \leqslant P$ and $\int_{0}^{t} \int_{0}^{t_{0}}|C(u+v)| d u d v \leqslant M$ imply that whenever $|\varphi(t)|<\delta$ on $\left[0, t_{0}\right]$ we have

$$
\begin{aligned}
\mid x\left(t+t_{0}, \varphi\right) & \leqslant P\left|\varphi\left(t_{0}\right)\right|+\delta \int_{0}^{t}|Z(t-u)| \int_{0}^{t_{0}}|C(u+v)| d v d u \\
& \leqslant P \delta+\delta P M<\varepsilon,
\end{aligned}
$$

provided that $\delta<\varepsilon /(P+P M)$. Hence, $x=0$ is uniformly stable. Thus, (viii) implies (ix).

Certainly, (ix) implies (x). 
Finally, if $x=0$ is stable, then $Z(t)$ is bounded so (x) implies (vii). This completes the proof of the theorem.

Now, we would like to extend Theorem 3 to the system

$$
x^{\prime}=A x+\int_{0}^{t} C(t, s) x(s) d s
$$

where $A$ and $C$ are $n \times n$ matrices, $n \geqslant 1, A$ constant, and $C$ is continuous for $0 \leqslant s \leqslant t<\infty$.

To this end, we are interested in finding an $n \times n$ symmetric matrix which satisfies the equation

$$
A^{T} B+B A=-I \text {. }
$$

It will be recognized that (3.6) is the standard equation from stability theory when $A$ is a stable matrix (cf. Hale [5, p. 295]). For if the characteristic roots of $A$ all have negative real parts, then (3.6) has a unique symmetric positive definite solution matrix $B$ and the Lyapunov function $V=x^{T} B x$ satisfies $V^{\prime}=-x^{T} x$ along any solution of $x^{\prime}=A x$.

It is immediate that if all characteristic roots of $A$ have positive real parts then (3.6) has a unique symmetric negative definite solution $B$.

Moreover, according to Barbashin [1, p. 1100], if $\lambda_{1}, \ldots, \lambda_{k}$ are the characteristic roots of $A$, then (3.6) has a unique symmetric solution provided that $\lambda_{i}+\lambda_{j} \neq 0$ for any $i$ and $j$.

It is easily shown that if $A$ has a characteristic root with zero real part then (3.6) has no solution.

One may construct examples of $A$ having $\lambda_{i}+\lambda_{j}=0$ and (3.6) having a solution, but not a unique one. Moreover, there are examples in which $\lambda_{i}+\lambda_{j}=0$ and (3.6) has no solution; however, in those same examples if a symmetric positive definite matrix $D$ is properly chosen then

$$
A^{T} B+B A=-D
$$

can be solved for $B$. In the following theorems equation $(3.6)^{\prime}$ is a satisfactory subsitute for (3.6).

THEOREM 8. Suppose (3.6) holds for some symmetric matrix $B$ and that there is a constant $M>0$ such that

$$
|B|\left(\int_{0}^{t}|C(t, s)| d s+\int_{t}^{\infty}|C(u, t)| d u\right) \leqslant M<1 .
$$

Then the zero solution of (3.5) is stable if and only if $B$ is positive definite.

Proof. We consider the functional

$$
V(t, x(\cdot))=x^{T} B x+|B| \int_{0}^{t} \int_{t}^{\infty}|C(u, s)| d u|x(s)|^{2} d s .
$$


Differentiate $V$ along a solution $x(t)$ of (3.5) to obtain

$$
\begin{aligned}
V_{(3.5)}^{\prime}(t, x(\cdot))= & {\left[x^{T} A^{T}+\int_{0}^{t} x^{T}(s) C^{T}(t, s) d s\right] B x+x^{T} B\left[A x+\int_{0}^{t} C(t, s) x(s) d s\right] } \\
& +|B| \int_{t}^{\infty}|C(u, t)| d u|x|^{2}-|B| \int_{0}^{t}|C(t, s)||x(s)|^{2} d s \\
= & -|x|^{2}+2 x^{T} B \int_{0}^{t} C(t, s) x(s) d s \\
& +|B| \int_{t}^{\infty}|C(u, t)| d u \cdot|x|^{2}-|B| \int_{0}^{t}|C(t, s)||x(s)|^{2} d s \\
\leqslant & -|x|^{2}+|B| \int_{0}^{t}|C(t, s)|\left(|x|^{2}+|x(s)|^{2}\right) d s \\
& +|B| \int_{t}^{\infty}|C(u, t)| d u|x|^{2}-|B| \int_{0}^{t}|C(t, s)||x(s)|^{2} d s \\
= & {\left[-1+|B|\left(\int_{0}^{t}|C(t, s)| d s+\int_{t}^{\infty}|C(u, s)| d u\right)\right]|x|^{2} } \\
\leqslant & {[-1+M]|x|^{2}=-\alpha|x|^{2} }
\end{aligned}
$$

where $\alpha=1-M>0$.

Now, if $B$ is positive definite, then $x^{T} B x>0$ for all $x \neq 0$ and hence $V(t, x(\cdot))$ is positive definite with $V_{(3.5)}^{\prime}(t, x(\cdot))$ negative definite. Thus, $x=0$ is stable.

Suppose that $x=0$ is stable but $B$ is not positive definite. Then there is an $x_{0} \neq 0$ with $x_{0}^{T} B x_{0} \leqslant 0$. If $x_{0}^{T} B x_{0}=0$, then along the solution $x\left(t, 0, x_{0}\right), V\left(0, x_{0}\right)=x_{0}^{T} B x_{0}$ $=0$ and $V^{\prime}(t, x(\cdot)) \leqslant-\alpha|x|^{2}$ so that for some $t_{1}>0$ we have $V\left(t_{1}, x(\cdot)\right)<0$. Thus $x^{T}\left(t_{1}\right) B x\left(t_{1}\right)<0$.

Hence, if $x^{T} B x$ is not always positive for $x \neq 0$, we may suppose there is an $x_{0} \neq 0$ with $x_{0}^{T} B x_{0}<0$.

Let $\varepsilon=1$ and $t_{0}=0$. Since $x=0$ is stable, there is a $\delta>0$ such that $\left|x_{0}\right|<\delta$ implies $\left|x\left(t, 0, x_{0}\right)\right|<1$ for $t \geqslant 0$. We may choose $x_{0}$ so that $\left|x_{0}\right|<\delta$ and $x_{0}^{T} B x_{0}<0$. Letting $x(t)=x\left(t, 0, x_{0}\right)$, we have

$$
\begin{aligned}
x^{T}(t) B x(t) & \leqslant V(t, x(\cdot)) \leqslant V\left(0, x_{0}\right)-\alpha \int_{0}^{t}|x(s)|^{2} d s \\
& \leqslant x_{0}^{T} B x_{0}-\alpha \int_{0}^{t}|x(s)|^{2} d s .
\end{aligned}
$$

We show that $x(t)$ is bounded away from zero. Suppose not; then there is a sequence $\left\{t_{n}\right\}$ tending to infinity monotonically such that $x\left(t_{n}\right) \rightarrow 0$. Hence, $x^{T}\left(t_{n}\right) B x\left(t_{n}\right) \rightarrow 0$, a contradiction to $x^{T}(t) B x(t) \leqslant x_{0}^{T} B x_{0}<0$.

Thus there is a $\gamma>0$ with $|x(t)|^{2} \geqslant \gamma$ so that $x^{T}(t) B x(t) \leqslant x_{0}^{T} B x_{0}-\alpha \gamma t$, implying that $|x(t)| \rightarrow \infty$ as $t \rightarrow \infty$. This contradicts $|x(t)|<1$ and completes the proof.

If $C(t, s)=C(t-s)$, then (3.5) reduces to

$$
x^{\prime}=A x+\int_{0}^{t} C(t-s) x(s) d s
$$

and (3.7) reduces to $2|B| \int_{0}^{\infty}|C(v)| d v<1$. We then have the following result. 
Corollary 3. Suppose (3.6) holds for some symmetric matrix B. If

$$
2|B|\left|\int_{0}^{\infty} C(v)\right| d v<1,
$$

then the zero solution of (3.8) is stable if and only if $B$ is positive definite.

We now prove a complete instability result which extends Corollary 2 to (3.5).

Let $B$ be a positive definite symmetric matrix satisfying

$$
A^{T} B+B A=I \text {. }
$$

THEOREM 9. Suppose (3.9) holds and

$$
|B|\left(\int_{0}^{t}|C(t, s)| d s+\int_{t}^{\infty}|C(u, t)| d u\right) \leqslant M<1 .
$$

Then the zero solution of (3.5) is completely unstable. Furthermore, for any $t_{0} \geqslant 0$ and any $\delta>0$ there is a continuous function $\varphi\left[0, t_{0}\right] \rightarrow R^{n}$ with $|\varphi(t)|<\delta$ and $\left|x\left(t, t_{0}, \varphi\right)\right| \geqslant\left[c_{1}+c_{2}\left(t-t_{0}\right)\right]^{1 / 2}$ for all $t \geqslant t_{0}$. Here, $c_{1}$ and $c_{2}$ are positive constants depending on $t_{0}$ and $\varphi$.

Proof. Consider the functional

$$
V(t, x(\cdot))=x^{T} B x-|B| \int_{0}^{t} \int_{t}^{\infty}|C(u, s)| d u|x(s)|^{2} d s
$$

and compute its derivative along the solution $x(t)=x\left(t, t_{0}, \varphi\right)$ of (3.5) so that

$$
\begin{aligned}
V_{(3.5)}^{\prime}(t, x(\cdot))= & {\left[x^{T} A^{T}+\int_{0}^{t} x^{T}(s) C^{T}(t, s) d s\right] B x+x^{T} B\left[A x+\int_{0}^{t} C(t, s) x(s) d s\right] } \\
& -|B| \int_{t}^{\infty}|C(u, t)| d u|x|^{2}+|B| \int_{0}^{t}|C(t, s)||x(s)|^{2} d s \\
= & x^{T} x+2 \int_{0}^{t} x^{T} B C(t, s) x(s) d s \\
& -|B| \int_{t}^{\infty}|C(u, t)| d u|x|^{2}+|B| \int_{0}^{t}|C(t, s)||x(s)|^{2} d s \\
\geqslant & |x|^{2}-2|B| \int_{0}^{t}|C(t, s)||x(s)||x| d s \\
& -|B| \int_{t}^{\infty}|C(u, t)| d u|x|^{2}+|B| \int_{0}^{t}|C(t, s)||x(s)|^{2} d s \\
\geqslant & |x|^{2}-|B| \int_{0}^{t}|C(t, s)|\left(|x|^{2}+|x(s)|^{2}\right) d s \\
& -|B| \int_{t}^{\infty}|C(u, t)| d u|x|^{2}+|B| \int_{0}^{t}|C(t, s)||x(s)|^{2} d s \\
\geqslant & {\left[1-|B|\left(\int_{0}^{t}|C(t, s)| d s+\int_{t}^{\infty}|C(u, t)| d u\right)\right]|x|^{2} } \\
\geqslant & {[1-M]|x|^{2}=\gamma|x|^{2} }
\end{aligned}
$$

where $\gamma=1-M>0$. 
Choose $\varphi$ on $\left[0, t_{0}\right]$ so that $V\left(t_{0}, \varphi(\cdot)\right)>0$. In particular, if $t_{0}=0$, then for any $\varphi(0) \neq 0$ we have $V(0, \varphi(\cdot))>0$. Then

$$
|B||x(t)|^{2} \geqslant x^{T}(t) B x(t) \geqslant V(t, x(\cdot)) \geqslant V\left(t_{0}, \varphi(\cdot)\right)+\gamma \int_{t_{0}}^{t}|x(s)|^{2} d s
$$

for all $t \geqslant t_{0}$. As $|x(t)|^{2} \geqslant V\left(t_{0}, \varphi(\cdot)\right) /|B|$, we have

$$
|B||x(t)|^{2} \geqslant V\left(t_{0}, \varphi(\cdot)\right)+\gamma V\left(t_{0}, \varphi(\cdot)\right)\left(t-t_{0}\right) /|B| .
$$

If $t_{0}=0$ and $\varphi(0) \neq 0$, then

$$
|x(t)|^{2} \geqslant\left[\varphi^{T}(0) B \varphi(0)\right] /|B|+\left(\gamma /|B|^{2}\right)\left[\varphi^{T}(0) B \varphi(0)\right] t .
$$

This is complete instability. The proof is now complete.

Select an $n \times n$ matrix $G(t)$ with

$$
\partial G(t) / \partial t=C(t)
$$

and set

$$
Q=A-G(0)
$$

so that (3.8) takes the form

$$
x^{\prime}=Q x+(d / d t) \int_{0}^{t} G(t-s) x(s) d s .
$$

Let $D$ be a symmetric matrix satisfying

$$
Q^{T} D+D Q=-I \text {. }
$$

If $D$ is any positive definite matrix, then there is a positive constant $k$ such that

$$
k|x|^{2} \leqslant x^{T} D x \text { for all } x .
$$

THEOREM 10. Suppose (3.12)-(3.15) hold. Let

(i) $2|D Q| \int_{0}^{\infty}|G(v)| d v<1$ and

(ii) $G(t) \rightarrow 0$ as $t \rightarrow \infty$.

Then the zero solution of (3.8) is stable if and only if $D$ is positive definite.

Proof. Consider the functional

$$
\begin{aligned}
V(t, x(\cdot))= & \left(x-\int_{0}^{t} G(t-s) x(s) d s\right)^{T} D\left(x-\int_{0}^{t} G(t-s) x(s) d s\right) \\
& +|D Q| \int_{0}^{t} \int_{t}^{\infty}|G(u-s)| d u|x(s)|^{2} d s,
\end{aligned}
$$

so that along a solution $x(t)$ of (3.14) we have

$$
\begin{aligned}
V_{(3.14)}^{\prime}(t, x(\cdot))= & x^{T} Q^{T} D\left(x-\int_{0}^{t} G(t-s) x(s) d s\right) \\
& +\left(x-\int_{0}^{t} G(t-s) x(s) d s\right)^{T} D Q x \\
& +|D Q| \int_{t}^{\infty}|G(u-t)| d u|x|^{2}-|D Q| \int_{0}^{t}|G(t-s)||x(s)|^{2} d s
\end{aligned}
$$




$$
\begin{aligned}
\leqslant & -|x|^{2}+|D Q| \int_{0}^{t}|G(t-s)|\left(|x|^{2}+|x(s)|^{2}\right) d s \\
& +|D Q| \int_{t}^{\infty}|G(u-t)||x|^{2}-|D Q| \int_{0}^{t}|G(t-s)||x(s)|^{2} d s \\
= & {\left[-1+|D Q|\left(\int_{0}^{t}|G(t-s)| d s+\int_{t}^{\infty}|G(u-t)| d u\right)\right]|x|^{2} } \\
\leqslant & {\left[-1+2|D Q| \int_{0}^{\infty}|G(v)| d v\right]|x|^{2} \stackrel{\text { def }}{=}-\mu|x|^{2} . }
\end{aligned}
$$

Suppose $D$ is positive definite and let $\varepsilon>0$ and $t_{0} \geqslant 0$ be given. We must find $\delta>0$ so that if $|\varphi(t)|<\delta$ on $\left[0, t_{0}\right]$, then $\left|x\left(t, t_{0}, \varphi\right)\right|<\varepsilon$ for $t \geqslant t_{0}$. As $V_{(3.14)}^{\prime}(t, x(\cdot)) \leqslant 0$, then

$$
\begin{aligned}
V(t, x(\cdot)) \leqslant & V\left(t_{0}, \varphi(\cdot)\right) \leqslant|D|\left(\left|\varphi\left(t_{0}\right)\right|+\int_{0}^{t_{0}}\left|G\left(t_{0}-s\right)\right||\varphi(s)| d s\right)^{2} \\
& +|D Q| \int_{0}^{t_{0}} \int_{t_{0}}^{\infty}|G(u-s)| d u|\varphi(s)|^{2} d s \leqslant \delta^{2} N^{2} \quad \text { for some } N>0 .
\end{aligned}
$$

Using (3.16) we have

$$
\begin{aligned}
V(t, x(\cdot)) & \geqslant\left(x-\int_{0}^{t} G(t-s) x(s) d s\right)^{T} D\left(x-\int_{0}^{t} G(t-s) x(s) d s\right) \\
& \geqslant k^{2}\left(|x|-\left|\int_{0}^{t} G(t-s) x(s) d s\right|\right)^{2}, \quad k \neq 0 .
\end{aligned}
$$

Thus,

$$
|x(t)| \leqslant(\delta N / k)+\int_{0}^{t}|G(t-s)||x(s)| d s .
$$

So long as $|x(t)|<\varepsilon$, we have $|x(t)|<(\delta N / k)+\varepsilon \int_{0}^{\infty}|G(v)| d v<\varepsilon$ for all $t \geqslant t_{0}$, provided that $\delta<(k / N)\left(1-\int_{0}^{\infty}|G(v)| d v\right) \varepsilon$. By (i) and the fact that $|2 D Q| \geqslant 1$, the right-hand side of the above inequality is positive. Hence, $x=0$ is stable.

Now, suppose that $x=0$ is stable but $D$ is not positive definite. Then it can be shown that there is an $x_{0} \neq 0$ with $x_{0}^{T} D x_{0}<0$ and $\left|x_{0}\right|<\delta$ for any given $\delta>0$. As $x=0$ is stable, we may choose $\delta$ so that $\left|x_{0}\right|<\delta$ implies $\left|x\left(t, 0, x_{0}\right)\right|<1$ for all $t \geqslant 0$.

Letting $x(t)=x\left(t, 0, x_{0}\right)$, we have

$$
V(t, x(\cdot)) \leqslant V\left(0, x_{0}\right)-\mu \int_{0}^{t}|x(s)|^{2} d s=-\eta-\mu \int_{0}^{t}|x(s)|^{2} d s,
$$

where $\eta=-x_{0}^{T} D x_{0}>0$. Thus,

$$
\begin{gathered}
\left(x(t)-\int_{0}^{t} G(t-s) x(s) d s\right)^{T} D\left(x(t)-\int_{0}^{t} G(t-s) x(s) d s\right) \\
\leqslant-\eta-\mu \int_{0}^{t}|x(s)|^{2} d s .
\end{gathered}
$$

Using the Schwarz inequality as in the proof of Theorem 4, we conclude that

$$
\left(\int_{0}^{t}|G(t-s)||x(s)| d s\right)^{2} \leqslant \int_{0}^{t}|G(t-s)| d s \int_{0}^{t}|G(t-s)||x(s)|^{2} d s .
$$


As $|x(t)|<1$ and $\int_{0}^{t}|G(t-s)| d s$ is bounded, then $\int_{0}^{t} G(t-s) x(s) d s$ is bounded. By (3.17) it follows that

$$
\eta+\mu \int_{0}^{t}|x(s)|^{2} d s \leqslant|D|\left(|x(t)|+\left|\int_{0}^{t} G(t-s) x(s) d s\right|\right)^{2} \leqslant K
$$

for some constant $K$. Thus, $|x(t)|^{2}$ is in $L^{1}[0, \infty)$. Now, $G(t) \rightarrow 0$ as $t \rightarrow \infty$ and $|x(t)|^{2}$ in $L^{1}$ imply that $\int_{0}^{t}|G(t-s)||x(s)|^{2} d s \rightarrow 0$ as $t \rightarrow \infty$. Thus, by the Schwarz inequality argument, $\int_{0}^{t} G(t-s) x(s) d s \rightarrow 0$ as $t \rightarrow \infty$.

By (3.17) we see that for large $t$ then $x^{T}(t) D x(t) \leqslant-\eta / 2$. Moreover, as $x \rightarrow 0$ we have $x^{T} D x \rightarrow 0$. Hence, we conclude that $|x(t)|^{2} \geqslant \gamma$ for some $\gamma>0$ and all $t$ sufficiently large. Thus, $\int_{0}^{t}|x(t)|^{2} d t \rightarrow \infty$ as $t \rightarrow \infty$, contradicting $|x(t)|^{2}$ being in $L^{\mathrm{l}}[0, \infty)$. Therefore, the assumption that $D$ is not positive definite is false and the proof is complete.

If

$$
\left|\int_{0}^{\infty} C(v) d v\right|<\infty
$$

we may define $G(t)$ by

$$
G(t)=-\int_{t}^{\infty} C(v) d v
$$

Thus,

$$
Q=A-G(0)=A+\int_{0}^{\infty} C(v) d v
$$

COROllary 4. Suppose (3.15) and (3.18)-(3.20) hold. Let

$$
2|D Q| \int_{0}^{\infty}\left|\int_{t}^{\infty} C(v) d v\right| d t<1 .
$$

Then the zero solution of (3.8) is stable if and only if $D$ is positive definite.

THEOREM 11. Suppose (3.15) and (3.18)-(3.20) hold. If

(i) $A+\int_{0}^{\infty} C(v) d v$ is a stable matrix and

(ii) $2|D Q| \int_{0}^{\infty}\left|\int_{t}^{\infty} C(v) d v\right| d t<1$

then the zero solution of (3.8) is stable and, furthermore, all solutions of (3.8) are in $L^{2}[0, \infty)$ and bounded.

If, in addition,

(iii) $\int_{0}^{\infty}|C(v)|^{2} d v<\infty$ or $\int_{0}^{\infty}|C(v)| d v<\infty$, then all solutions of (3.8) tend to zero as $t \rightarrow \infty$ and, hence, the zero solution of (3.8) is asymptotically stable.

If, in addition,

(iv) $\int_{0}^{\infty} \int_{t}^{\infty}|C(v)| d v d t<\infty$,

then all solutions of (3.8) are in $L^{1}[0, \infty)$ and the zero solution of (3.8) is uniformly asymptotically stable.

Proof. Let (i) and (ii) hold. Then $D$ is positive definite and by Corollary $4, x=0$ is stable; hence, all solutions are bounded. 
Using the functional $V(t, x(\cdot))$ in the proof of Theorem 10 we have

$$
V_{(3.14)}^{\prime}(t, x(\cdot)) \leqslant-\mu|x(t)|^{2}
$$

for $\mu>0$ and $t \geqslant t_{0}$. Thus, $|x(t)|^{2}$ is in $L^{1}[0, \infty)$.

If, in addition, (iii) holds, then (3.8) yields

$$
\begin{aligned}
\left|x^{\prime}(t)\right| & \leqslant|A||x(t)|+\int_{0}^{t}|C(t-s)||x(s)| d s \\
& \leqslant|A||x(t)|+(1 / 2) \int_{0}^{t}|C(t-s)|^{2} d s+(1 / 2) \int_{0}^{t}|x(s)|^{2} d s .
\end{aligned}
$$

Thus, $\left|x^{\prime}(t)\right|$ is bounded and so is $\left(|x(t)|^{2}\right)^{\prime}$; for,

$$
\left(|x(t)|^{2}\right)^{\prime}=\left(x^{T}(t) x(t)\right)^{\prime} \leqslant 2|x(t)|\left|x^{\prime}(t)\right| .
$$

Hence, $x(t) \rightarrow 0$ as $t \rightarrow \infty$ and $x=0$ is asymptotically stable.

Suppose (iv) holds. Since (iv) is equivalent to (3.3) and since all solutions of (3.8) tend to zero as $t \rightarrow \infty$, we may apply Theorem 7 to conclude that the zero solution of (3.8) is uniformly asymptotically stable and all solutions of (3.8) are in $L^{1}[0, \infty)$.

Corollary 5. Suppose (3.15) and (3.20) hold. If

(i) $A+\int_{0}^{\infty} C(v) d v$ is a stable matrix and

(ii) $2|D Q| \int_{0}^{\infty} \int_{t}^{\infty}|C(v)| d v d t<1$,

then the zero solution of (3.8) is uniformly asymptotically stable.

Proof. This is an immediate consequence of Theorem 11 as, by (i) and (ii), all conditions of the theorem are satisfied.

If we now return to the scalar equation (2.7), we deduce from Theorem 11 the following stability criterion.

THEOREM 12. Suppose that

(i) $\int_{0}^{\infty} \int_{t}^{\infty}|C(v)| d v d t<\infty$,

(ii) $\int_{0}^{\infty}\left|\int_{t}^{\infty} C(v) d v\right| d t<1$, and

(iii) $A+\int_{0}^{\infty} C(v) d v<0$.

Then the zero solution of (2.7) is uniformly asymptotically stable.

Theorem 12 may now be compared with Brauer's result (b). While (ii) is sufficiently mild as we have seen from Example 2, Brauer's basic assumption (2.4) implies (i). For, using integration by parts, we have

$$
\begin{aligned}
\int_{0}^{\infty} t|C(t)| d t & \geqslant \int_{0}^{t} s|C(s)| d s=-t \int_{t}^{\infty}|C(v)| d v+\int_{0}^{t} \int_{s}^{\infty}|C(v)| d v d s \\
& \geqslant-\int_{0}^{\infty} t|C(t)| d t+\int_{0}^{t} \int_{s}^{\infty}|C(v)| d v d s .
\end{aligned}
$$

Thus,

$$
\int_{0}^{t} \int_{s}^{\infty}|C(v)| d v d s \leqslant 2 \int_{0}^{\infty} t|C(t)| d t<\infty .
$$

Also, Brauer's result applies only to a limited class of functions and fails to specify how small the quantity $\int_{0}^{\infty} t|C(t)| d t$ must be in order to insure uniform asymptotic stability. Our result, however, is quite specific, has a wider range of applications, and nicely extends to the system (3.8) in the form of Theorems 10 and 11. 
The next result is concerned with the asymptotic behavior of solutions of the system (3.8).

Letting $C^{(0)}(t)=C(t)$ and $C^{(i)}(t)=d^{i} C(t) / d t^{i}$ for $i=0,1,2, \ldots$, we prove the following result.

THEOREM 13. Suppose (3.15) and (3.18)-(3.20) hold. Suppose also that $C^{(m-1)}(t)$ is continuous for some positive integer $m$. If

(i) $A+\int_{0}^{\infty} C(v) d v$ is a stable matrix,

(ii) $2|D Q| \int_{0}^{\infty}\left|\int_{t}^{\infty} C(v) d v\right| d t<1$, and

(iii) $\int_{0}^{\infty}\left|C^{(i)}(v)\right| d v<\infty, i=0,1, \ldots, m-1$,

then the zero solution of (3.8) is asymptotically stable. Furthermore, every solution $x(t)=x\left(t, t_{0}, \varphi\right)$ of (3.8) has the property that $x^{(i)}(t) \rightarrow 0$ as $t \rightarrow \infty, i=0,1, \ldots, m$, and $x^{(i)}(t)$ is in $L^{2}\left[t_{0}, \infty\right)$ for $i=0,1, \ldots, m$.

Proof. The asymptotic stability of $x=0$ follows from Theorem 11 . We prove the last part by induction.

For $m=1$, use (3.8) to obtain

$$
\left|x^{\prime}(t)\right| \leqslant|A||x(t)|+\int_{0}^{t}|C(t-s)||x(s)| d s .
$$

Now, (i)-(iii) imply, by Theorem 10 , that $x(t) \rightarrow 0$ and $x(t)$ is in $L^{2}\left[t_{0}, \infty\right)$. As $C(t)$ is in $L^{1}$, that integral tends to zero; hence, $x^{\prime}(t) \rightarrow 0$ as $t \rightarrow \infty$. Furthermore, the integral is the convolution of an $L^{1}$ function with an $L^{2}$ function and, therefore, is an $L^{2}$ function (cf. Hewitt and Stromberg [6, p. 397]).

We now assume that the theorem is true for $m=k$ and show it is true for $m=k+1, k=1,2,3, \ldots$. Differentiate (3.8) $k$ times and obtain

$$
\begin{aligned}
\left|x^{(k+1)}(t)\right| \leqslant & |A|\left|x^{(k)}(t)\right|+\sum_{i=0}^{k-1}\left|C^{(i)}(0)\right|\left|x^{(k-1-i)}(t)\right| \\
& +\int_{0}^{t}\left|C^{(k)}(t-s)\right||x(s)| d s .
\end{aligned}
$$

We assume (iii) holds for $m=k+1$. Then the above integral is the convolution of an $L^{1}$ function with a function tending to zero. Thus, the integral tends to zero and since the theorem is true for $m=k$, then $x^{(i)}(t) \rightarrow 0$ as $t \rightarrow \infty, i=0,1, \ldots, k$, and hence $x^{(k+1)}(t) \rightarrow 0$ as $t \rightarrow \infty$. That is, $x^{(i)} t \rightarrow 0$ as $t \rightarrow \infty$ for $i=0,1, \ldots, k+1$.

Also, $x^{(i)}(t)$ is in $L^{2}$ for $i=0,1, \ldots, k$ and the integral above is the convolution of an $L^{1}$ function with an $L^{2}$ function. Thus, the integral is an $L^{2}$ function. Hence, $x^{(k+1)}(t)$ is in $L^{2}\left[t_{0}, \infty\right)$; that is, $x^{(i)}(t)$ is in $L^{2}\left[t_{0}, \infty\right)$ for $i=0,1, \ldots, k+1$. This completes the proof.

4. Perturbation. There are two interesting perturbations of (3.5) that we wish to consider in this section. We first consider the system

$$
\text { (4.1) } x^{\prime}=A x+A_{1}(t) x+H_{1}(x) x+\int_{0}^{t}\left[C(t, s)+C_{1}(t, s) H_{2}(x(s))\right] x(s) d s
$$

where $A, A_{1}, C, C_{1}, H_{1}$, and $H_{2}$ are $n \times n$ matrices, $A$ is constant, $A_{1}(t)$ is continuous for $0 \leqslant t<\infty, C(t, s)$ and $C_{1}(t, s)$ are continuous for $0 \leqslant s \leqslant t<\infty$, and $H_{1}(x)$ 
and $H_{2}(x)$ are continuous on some open set $U \subset R^{n}$ with $0 \in U$. We assume there are positive constants $m$ and $J$ such that

$$
\left|A_{1}(t)\right| \leqslant m \text { and }\left|C_{1}(t, s)\right| \leqslant J|C(t, s)|
$$

and

$$
H_{i}(0)=0, \quad i=1,2 \text {. }
$$

Also, we suppose there is a symmetric matrix $B$ with

$$
A^{T} B+B A=-I \text {. }
$$

THEOREM 14. Suppose (4.2)-(4.4) hold and there is a constant $M>0$ with

$$
|B|\left(\int_{0}^{t}|C(t, s)| d s+\int_{0}^{\infty}|C(u, t)| d u\right) \leqslant M<1 .
$$

Then for $m$ sufficiently small, the zero solution of (4.1) is stable if and only if $B$ is positive definite.

Proof. Consider the functional

$$
V(t, x(\cdot))=x^{T} B x+K \int_{0}^{t} \int_{t}^{\infty}|C(u, s)| d u|x(s)|^{2} d s
$$

so that along a solution $x(t)=x\left(t, t_{0}, \varphi\right)$ of (4.1) we have

$$
\begin{aligned}
V_{(4.1)}^{\prime}(t, x(\cdot))= & \left(x^{T} A^{T}+x^{T} A_{1}^{T}+x^{T} H_{1}^{T}\right. \\
& \left.+\int_{0}^{t} x^{T}(s)\left[C^{T}(t, s)+H_{2}^{T}(x(s)) C_{1}^{T}(t, s)\right] d s\right) B x \\
& +x^{T} B\left(A x+A_{1} x+H_{1} x+\int_{0}^{t}\left[C(t, s)+C_{1}(t, s) H_{2}(x(s))\right] x(s) d s\right) \\
& +K \int_{t}^{\infty}|C(u, t)| d u|x|^{2}-K \int_{0}^{t}|C(t, s)||x(s)|^{2} d s \\
\leqslant & -|x|^{2}+2|B|\left|A_{1}(t)\right||x|^{2}+2|B|\left|H_{1}(x)\right||x|^{2} \\
& +|B| \int_{0}^{t}\left(|C(t, s)|+\left|C_{1}(t, s)\right|\left|H_{2}(x(s))\right|\right)\left(|x(s)|^{2}+|x|^{2}\right) d s \\
& +K \int_{t}^{\infty}|C(u, t)| d u|x|^{2}-K \int_{0}^{t}|C(t, s)||x(s)|^{2} d s \\
\leqslant & -|x|^{2}+2|B| m|x|^{2}+2|B|\left|H_{1}(x) \| x\right|^{2} \\
& +|B| \int_{0}^{t}\left(|C(t, s)|+J|C(t, s)|\left|H_{2}(x(s))\right|\right)\left(|x(s)|^{2}+|x|^{2}\right) d s \\
& +K \int_{t}^{\infty}|C(u, t)| d u|x|^{2}-K \int_{0}^{t}|C(t, s) \| x(s)|^{2} d s
\end{aligned}
$$




$$
\begin{aligned}
& \leqslant\left[-1+|B|\left(2 m+2\left|H_{1}(x)\right|\right.\right. \\
& \left.\quad+\int_{0}^{t}|C(t, s)|\left(1+J\left|H_{2}(x(s))\right|\right) d s\right) \\
& \left.\quad+K \int_{t}^{\infty}|C(u, t)| d u\right]|x|^{2} \\
& +|B| \int_{0}^{t}|C(t, s)|\left(1+J\left|H_{2}(x(s))\right|\right)|x(s)|^{2} d s \\
& \quad-K \int_{0}^{t}|C(t, s)||x(s)|^{2} d s .
\end{aligned}
$$

Now $H_{i}(x)$ are continuous with $H_{i}(0)=0$. Thus, for each $\eta>0$ there is a $\gamma>0$ such that $|x| \leqslant \gamma$ implies $\left|H_{i}(x)\right|<\eta, i=0,1$. Therefore,

$$
\begin{aligned}
V_{(4.1)}^{\prime}(t, x(\cdot)) \leqslant & {\left[-1+|B|\left(2 m+2 \eta+\int_{0}^{t}|C(t, s)|(1+J \eta) d s\right)\right.} \\
& \left.\quad+K \int_{t}^{\infty}|C(u, t)| d u\right]|x|^{2} \\
& +|B| \int_{0}^{t}|C(t, s)|(1+J \eta)|x(s)|^{2} d s-K \int_{0}^{t}|C(t, s)||x(s)|^{2} d s .
\end{aligned}
$$

Letting $K=|B|(1+J \eta)$, we have

$$
\begin{aligned}
V_{(4.1)}^{\prime}(t, x(\cdot)) \leqslant & \leqslant-1+2|B|(m+\eta) \\
& \left.\quad+|B|(1+J \eta)\left(\int_{0}^{t}|C(t, s)| d s+\int_{t}^{\infty}|C(u, t)| d u\right)\right]|x|^{2} \\
& \leqslant[-1+2|B|(m+\eta)+M(1+J \eta)]|x|^{2} .
\end{aligned}
$$

Let $\alpha=1-2|B|(m+\eta)-M(1+J \eta)$ and choose $m$ and $\eta$ sufficiently small so that $\alpha>0$. Thus, $V_{(4.1)}^{\prime}(t, x(\cdot)) \leqslant-\alpha|x|^{2}$ so long as $|x(s)| \leqslant \gamma$ for $0 \leqslant s \leqslant t$.

Suppose that $B$ is positive definite and let $\varepsilon>0$ and $t_{0} \geqslant 0$ be given. Assume $\varepsilon<\gamma$ and find $\delta>0$ such that if $|\varphi(t)|<\delta$ on $\left[0, t_{0}\right]$, then $\left|x\left(t, t_{0}, \varphi\right)\right|<\varepsilon$ for all $t \geqslant t_{0}$.

Since $B$ is positive definite, then by (3.14) there is a $k \neq 0$ such that

$$
\begin{aligned}
k^{2}|x|^{2} & \leqslant x^{T} B x \leqslant V(t, x(\cdot)) \leqslant V\left(t_{0}, \varphi(\cdot)\right) \\
& \leqslant|B|\left|\varphi\left(t_{0}\right)\right|^{2}+|B|(1+J \eta) \int_{0}^{t_{0}} \int_{t_{0}}^{\infty}|C(u, s)| d u|\varphi(s)|^{2} d s<\delta^{2} N^{2}
\end{aligned}
$$

where

$$
N^{2}=|B|\left[1+(1+J \eta) \int_{0}^{t_{0}} \int_{t_{0}}^{\infty}|C(u, s)| d u d s\right] .
$$

Thus $|x(t)|<\varepsilon$ if $\delta<k \varepsilon / N$ for all $t \geqslant t_{0}$. Hence, $x=0$ is stable.

Suppose now that $x=0$ is stable but $B$ is not positive definite. Then we may argue as in the proof of Theorem 8 to conclude that if $\varepsilon=\gamma$ and $t_{0} \geqslant 0$, there is a 
$\delta>0$ and $x_{0} \neq 0$ with $\left|x_{0}\right|<\delta$ and $x_{0}^{T} B x_{0}<0$. Continuing the argument we find $t_{1}>0$ such that $\left|x\left(t, 0, x_{0}\right)\right|=\gamma$. This will complete the proof.

We now consider another interesting form of perturbation of (3.5), namely the system

$$
x^{\prime}=A x+f(t, x)+\int_{0}^{t} C(t, s) x(s) d s
$$

in which $A$ and $C$ are $n \times n$ matrices, $A$ constant and $C(t, s)$ continuous for $0 \leqslant s \leqslant t<\infty$. Also, $f:[0, \infty) \times R^{n} \rightarrow R^{n}$ is continuous with

$$
|f(t, x)| \leqslant \lambda(t)(|x|+1)
$$

with $\lambda:[0, \infty) \rightarrow[0, \infty)$ continuous and $\int_{0}^{\infty} \lambda(s) d s<\infty$ and

$$
\lambda(t) \rightarrow 0 \text { as } t \rightarrow \infty \text {. }
$$

We also assume there is a symmetric matrix $B$ with

$$
A^{T} B+B A=-I \text {. }
$$

THEOREM 15. Suppose (4.7)-(4.9) hold and there is a constant $M>0$ such that

$$
|B|\left(\int_{0}^{t}|C(t, s)| d s+\int_{t}^{\infty}|C(u, t)| d u\right) \leqslant M<1 .
$$

Then all solutions of (4.6) are bounded if and only if $B$ is positive definite.

Proof. Consider the functional

$$
V(t, x(\cdot))=\left[x^{T} B x+1+|B| \int_{0}^{t} \int_{t}^{\infty}|C(u, s)| d u|x(s)|^{2} d s\right] e^{-L \int_{0}^{t} \lambda(s) d s}
$$

where $L$ is a positive constant.

We differentiate $V$ along a solution $x(t)=x\left(t, t_{0}, \varphi\right)$ of (4.6) to obtain

$$
\begin{aligned}
& V_{(4.6)}^{\prime}(t, x(\cdot)) \\
& =-L \lambda(t) V+\left[\left(x^{T} A^{T}+f^{T}(t, x)+\int_{0}^{t} x^{T}(s) C^{T}(t, s) d s\right) B x\right. \\
& +x^{T} B\left(A x+f(t, x)+\int_{0}^{t} C(t, s) x(s) d s\right) \\
& \left.+|B| \int_{t}^{\infty}|C(u, t)| d u|x|^{2}-|B| \int_{0}^{t}|C(t, s)||x(s)|^{2} d s\right] e^{-L \int_{0}^{t} \lambda(s) d s} \\
& \leqslant-L \lambda(t) V+\left\{\left[-1+|B|\left(\int_{0}^{t}|C(t, s)| d s+\int_{t}^{\infty}|C(u, t)| d u\right)\right]|x|^{2}\right. \\
& +2|B||x \| f(t, x)|\} e^{-L \int_{0}^{t} \lambda(s) d s} \\
& \leqslant-L \lambda(t) V+\left[(-1+M)|x|^{2}+2|B||x| \lambda(t)(|x|+1)\right] e^{-L \int_{0}^{t} \lambda(s) d s} \\
& \leqslant e^{-L \int_{0}^{t} \lambda(s) d s}\left[-L \lambda(t)+(-1+M+2|B| \lambda(t))|x|^{2}+2|B| \lambda(t)|x|\right] \\
& \leqslant e^{-L \int_{0}^{t} \lambda(s) d s}\left[-L \lambda(t)+(-1+M+2|B| \lambda(t))|x|^{2}+|B| \lambda(t)+|B| \lambda(t)|x|^{2}\right] .
\end{aligned}
$$


Letting $L=|B|$ and taking $S$ sufficiently large so that $-1+M+3|B| \lambda(t) \leqslant-\beta$ for some $\beta>0$ and all $t \geqslant S$, we find

$$
V_{(4.6)}^{\prime}(t, x(\cdot)) \leqslant e^{-L \int_{0}^{t} \lambda(s) d s}\left(-\beta|x|^{2}\right) \leqslant-\gamma|x|^{2}
$$

for some $\gamma>0$ and all $t \geqslant S$.

Suppose that $B$ is positive definite and $x(t)$ is a solution of (4.6). Then by the growth condition on $f, x(t)$ can be continued for all future time. Hence, for $t \geqslant S$ we have $V(t, x(\cdot)) \leqslant V(S, x(\cdot))$ so that $x(t)$ is bounded.

Suppose that all solutions are bounded but $B$ is not positive definite. We may now argue as in the proof of Theorem 8 to conclude that there is an $x_{0} \neq 0$ with $x_{0}^{T} B x_{0}<0$. Take $t_{0}=S$ and select a continuous function $\varphi$ on $\left[0, t_{0}\right]$ with $V\left(t_{0}, \varphi(\cdot)\right)<0$.

Since $V_{(4.6)}^{\prime}(t, x(\cdot)) \leqslant-\gamma|x(t)|^{2}$ for $t \geqslant S$, we have

$$
V(t, x(\cdot)) \leqslant V(S, \varphi(\cdot))-\gamma \int_{S}^{t}|x(u)|^{2} d u .
$$

We may argue as in the proof of Theorem 8 to conclude that $x(t)=x\left(t, t_{0}, \varphi\right)$ is bounded away from zero and hence $V(t, x(\cdot)) \rightarrow-\infty$ as $t \rightarrow \infty$. Thus, $x(t)$ is unbounded, a contradiction. This completes the proof.

5. Asymptotic behavior. We propose to extend Theorem 13 to a nonconvolution equation. For simplicity we discuss the scalar equation

$$
x^{\prime}=A(t) x+\int_{0}^{t} C(t, s) x(s) d s
$$

where $A$ is continuous for $0 \leqslant t<\infty$ and $C$ is continuous for $0 \leqslant s \leqslant t<\infty$.

Assume for the present time that $A(t)$ and $C(t, s)$ are continuously differentiable and that both

$$
\int_{t}^{\infty}|C(u, s)| d u \text { and } \int_{t}^{\infty}[\partial C(u, t) / \partial t] d u \text { exist. }
$$

Define $G(t, s)$ and $Q(t)$, respectively, by

$$
G(t, s)=-\int_{t}^{\infty} C(u, s) d u
$$

and

$$
Q(t)=A(t)-G(t, t)
$$

so that (5.1) may be written as

$$
x^{\prime}=Q(t) x+(d / d t) \int_{0}^{t} G(t, s) x(s) d s .
$$

THEOREM 16. Suppose (5.2)-(5.4) hold. If there are constants $Q_{1}, Q_{2}, J, N$, and $R$ with $R<2$ such that for $0 \leqslant t<\infty$ we have

(i) $-Q_{2} \leqslant Q(t) \leqslant-Q_{1}<0$,

(ii) $\int_{0}^{t}|G(t, s)| d s \leqslant J<1$,

(iii) $\int_{0}^{t}|G(t, s)| d s+\int_{t}^{\infty}|G(u, t)| d u \leqslant R Q_{1} / Q_{2}$, and

(iv) $\int_{0}^{t}|C(t, s)| d s+\left|\int_{t}^{\infty} C(u, t) d u\right| \leqslant N$,

then the zero solution of (5.1) is asymptotically stable. Furthermore, every solution $x(t)=x\left(t, t_{0}, \varphi\right)$ of $(5.1)$ is in $L^{2}\left[t_{0}, \infty\right)$. 
In addition, if

(v) $\left|Q^{\prime}(t)-Q(t) G(t, t)\right|+|Q(t)| \int_{0}^{t}|C(t, s)| d s \leqslant R Q_{1}$,

(vi) $C(t, t), \int_{0}^{t}|\partial C(t, s) / \partial t| d s$, and $\int_{t}^{\infty}[\partial C(u, t) / \partial t] d u$ are bounded, then $x^{(i)}(t) \rightarrow 0$ as $t \rightarrow \infty$ and $x^{(i)}(t)$ are in $L^{2}\left[t_{0}, \infty\right)$ for $i=0,1$.

Proof. The stability of $x=0$ follows from Theorem 4 . Now, using the functional $V(t, x(\cdot))$ as in the proof of Theorem 4, we obtain $V^{\prime}(t, x(\cdot)) \leqslant-\beta x^{2}(t)$ for some $\beta>0$ and all $t \geqslant t_{0}$. As $V(t, x(\cdot)) \geqslant 0$, we have $x(t)$ in $L^{2}\left[t_{0}, \infty\right)$. To show that $x=0$ is asymptotically stable we first observe from (5.3) and (iv) that $G(t, t)$ is bounded and since $Q(t)$ is bounded, then it follows from (5.4) that $A(t)$ is bounded. Now

$$
\left|x^{\prime}(t)\right| \leqslant|A(t)||x(t)|+\int_{0}^{t}|C(t, s) \| x(s)| d s
$$

yields, using (iv), that $x^{\prime}(t)$ is bounded. Since $x^{2}(t)$ is $L^{1}\left[t_{0}, \infty\right)$ and $\left[x^{2}(t)\right]^{\prime}=$ $2 x(t) x^{\prime}(t)$ is bounded, it follows that $x(t) \rightarrow 0$ as $t \rightarrow \infty$. Hence, $x=0$ is asymptotically stable.

To show that $x^{\prime}(t)$ is in $L^{2}\left[t_{0}, \infty\right)$, we differentiate (5.5) obtaining

$$
x^{\prime \prime}=(Q(t) x)^{\prime}+(d / d t)\left[G(t, t) x+\int_{0}^{t} C(t, s) x(s) d s\right] .
$$

Let $Q=Q(t), G=G(t, t)$ and consider the functional

$$
\begin{aligned}
W\left(t, x(\cdot), x^{\prime}(\cdot)\right)= & \left(x^{\prime}-\left[G x+\int_{0}^{t} C(t, s) x(s) d s\right]\right)^{2} \\
& +K \int_{t}^{\infty} x^{2}(s) d s+M \int_{0}^{t} \int_{t}^{\infty}|C(u, s)| d u x^{2}(x) d s
\end{aligned}
$$

whose derivative along a solution $x(t)=x\left(t, t_{0}, \varphi\right)$ for $t \geqslant t_{0}$ satisfies

$$
\begin{aligned}
W^{\prime}\left(t, x(\cdot), x^{\prime}(\cdot)\right)= & 2\left(x^{\prime}-\left[G x+\int_{0}^{t} C(t, s) x(s) d s\right]\right)(Q x)^{\prime}-K x^{2} \\
& +M \int_{t}^{\infty}|C(u, t)| d u x^{2}-M \int_{0}^{t}|C(t, s)| x^{2}(s) d s \\
= & 2 Q\left(x^{\prime}\right)^{2}+2\left(Q^{\prime} x-Q\left[G x+\int_{0}^{t} C(t, s) x(s) d s\right]\right) x^{\prime} \\
& -2\left(G x+\int_{0}^{t} C(t, s) x(s) d s\right) Q^{\prime} x-K x^{2} \\
& +M \int_{t}^{\infty}|C(u, t)| d u x^{2}-M \int_{0}^{t}|C(t, s)| x^{2}(s) d s \\
\leqslant & 2 Q\left(x^{\prime}\right)^{2}+2\left|Q^{\prime}-Q G\right||x|\left|x^{\prime}\right| \\
& +2|Q| \int_{0}^{t}|C(t, s)|\left|x(s) \| x^{\prime}\right| d s+2\left|G Q^{\prime}\right| x^{2} \\
& +2\left|Q^{\prime}\right| \int_{0}^{t}|C(t, s)||x(s)||x| d s-K x^{2} \\
& +M \int_{t}^{\infty}|C(u, t)| d u x^{2}-M \int_{0}^{t}|C(t, s)| x^{2}(s) d s
\end{aligned}
$$




$$
\begin{aligned}
\leqslant & 2 Q\left(x^{\prime}\right)^{2}+\left|Q^{\prime}-Q G\right|\left(x^{2}+x^{\prime 2}\right) \\
& +|Q| \int_{0}^{t}|C(t, s)|\left(x^{2}(s)+\left(x^{\prime}\right)^{2}\right) d s \\
& +2\left|G Q^{\prime}\right| x^{2}+\left|Q^{\prime}\right| \int_{0}^{t}|C(t, s)|\left(x^{2}(s)+x^{2}\right) d s \\
& -K x^{2}+M \int_{t}^{\infty}|C(u, t)| d u x^{2}-M \int_{0}^{t}|C(t, s)| x^{2}(s) d s \\
\leqslant & {\left[2 Q+\left|Q^{\prime}-Q G\right|+|Q| \int_{0}^{t}|C(t, s)| d s\right]\left(x^{\prime}\right)^{2} } \\
& +\left[\left|Q^{\prime}-Q G\right|+2\left|G Q^{\prime}\right|+\left|Q^{\prime}\right| \int_{0}^{t}|C(t, s)| d s\right. \\
& \left.+M \int_{t}^{\infty}|C(u, s)| d u-K\right] x^{2} \\
& +\left(|Q|+\left|Q^{\prime}\right|-M\right) \int_{0}^{t}|C(t, s)| x^{2}(s) d s .
\end{aligned}
$$

By (v), $Q^{\prime}(t)$ is bounded. Thus, by choosing $K$ and $M$ sufficiently large we obtain

$$
\begin{aligned}
W^{\prime}\left(t, x(\cdot), x^{\prime}(\cdot)\right) & \leqslant\left[-2 Q_{1}+\left|Q^{\prime}-Q G\right|+|Q| \int_{0}^{t}|C(t, s)| d s\right]\left(x^{\prime}\right)^{2} \\
& \leqslant\left[-2 Q_{1}+R Q_{1}\right]\left(x^{\prime}\right)^{2} \stackrel{\text { def }}{=}-\gamma\left(x^{\prime}\right)^{2}
\end{aligned}
$$

As $W\left(t, x(\cdot), x^{\prime}(\cdot)\right) \geqslant 0$, we have $x^{\prime}(t)$ in $L^{2}\left[t_{0}, \infty\right)$. To show $x^{\prime}(t) \rightarrow 0$, we differentiate $(5.1)$ and obtain

$$
x^{\prime \prime}(t)=A(t) x^{\prime}(t)+A^{\prime}(t) x(t)+C(t, t) x(t)+\int_{0}^{t}[\partial C(t, s) / \partial t] x(s) d s .
$$

Since $Q(t)=A(t)-G(t, t)=A(t)+\int_{t}^{\infty} C(u, t) d u$, we have $Q^{\prime}(t)=A^{\prime}(t)-$ $C(t, t)+\int_{t}^{\infty}[\partial C(u, t) / \partial t] d u$. Thus, by (v) and (vi), $A^{\prime}(t)$ is bounded and hence by (5.6) and (5.8), $x^{\prime \prime}(t)$ is bounded; therefore, $\left(\left(x^{\prime}\right)^{2}\right)^{\prime}=2 x^{\prime} x^{\prime \prime}$ is also bounded. As $\left(x^{\prime}\right)^{2}$ is in $L^{1}\left[t_{0}, \infty\right)$, we have $x^{\prime}(t) \rightarrow 0$ as $t \rightarrow \infty$. This completes the proof.

It is worth pointing out that when $A(t)=A=$ constant and $C(t, s)=C(t-s)$, the conditions (i)-(v) of Theorem 16 reduce to (i)-(iii) of Theorem 13 when $n=m=1$.

We now consider the general vector case and assume that $A(t)$ and $C(t, s)$ are $n$ times continuously differentiable and that

$$
\int_{t}^{\infty}\left|\partial^{i-1} C(u, s) / \partial u^{i-1}\right| d u \text { and } \int_{t}^{\infty}\left[\partial^{i} C(u, t) / \partial t^{i}\right] d u \text { exist }
$$

for $i=1,2, \ldots, n$.

Let $C_{t^{i}}$ and $C_{s^{i}}$ denote the $i$ th derivative of $C(t, t)$ with respect to the first and second arguments, respectively. Let $C^{(i)}=\left[d^{i} C(t, t) / d t^{i}\right]$ and $C^{(0)}=C_{t^{0}}=C_{s^{0}}=$ $C(t, t)$. Then $C^{(i)}, C_{t^{i}}$, and $C_{s^{i}}$ are defined for $i=0,1, \ldots, n$. 
THEOREM 17. Let (5.3), (5.4), and (5.9) hold. Suppose also that (i)-(iv) of Theorem 16 as well as the following conditions are satisfied:

(a)

$$
\begin{gathered}
\sum_{i=1}^{k}\left|\left(\begin{array}{c}
k \\
i
\end{array}\right) Q^{(i)}(t)-Q(t)\left[\left(\begin{array}{c}
k-1 \\
i-1
\end{array}\right) G^{(i-1)}(t, t)+\sum_{j=2}^{i}\left(\begin{array}{c}
k-j \\
i-j
\end{array}\right) C_{t^{j-2}}^{(i-j)}(t, t)\right]\right| \\
+|Q(t)| \int_{0}^{t}\left|\partial^{k-1} C(t, s) / \partial t^{k-1}\right| d s \leqslant R Q_{1},
\end{gathered}
$$

for $k=1,2, \ldots, n$, and

(b)

$$
C^{(k-1)}(t, t), \quad \int_{0}^{t}\left|\partial^{k} C(t, s) / \partial t^{k}\right| d s \quad \text { and } \quad \int_{t}^{\infty}\left[\partial^{k} C(u, t) / \partial t^{k}\right] d u
$$

are bounded for $k=1,2, \ldots, n$.

Then the zero solution of (5.1) is asymptotically stable and, furthermore, every solution $x(t)=x\left(t, t_{0}, \varphi\right)$ of $(5.1)$ has the property that $x^{(i)}(t) \rightarrow 0$ as $t \rightarrow \infty$ and $x^{(i)}(t)$ is in $L^{2}\left[t_{0}, \infty\right)$ for $i=0,1, \ldots, n$.

Before proving the theorem we establish the following identity:

$$
\sum_{j=0}^{k-2}\left(C_{t^{j}} x\right)^{(k-2-j)}=\sum_{i=1}^{k} \sum_{j=2}^{i}\left(\begin{array}{c}
k-j \\
i-j
\end{array}\right) C_{t^{\prime}-2}^{(i-j)} x^{(k-i)},
$$

for $k=2,3, \ldots, n$.

Using the product rule

we have

$$
(u v)^{(m)}=\sum_{i=0}^{m}\left(\begin{array}{c}
m \\
i
\end{array}\right) u^{(i)} v^{(m-i)}
$$

$$
\begin{aligned}
\left(C_{t^{j}} x\right)^{(k-2-j)} & =\sum_{i=0}^{k-2-j}\left(\begin{array}{c}
k-2-j \\
i
\end{array}\right) C_{t^{\prime}}^{(i)} x^{(k-2-j-i)} \\
& =\sum_{i=j+2}^{k}\left(\begin{array}{c}
k-2-j \\
i-2-j
\end{array}\right) C_{t^{j}}^{(i-2-j)} x^{(k-i)}
\end{aligned}
$$

Thus,

$$
\sum_{j=0}^{k-2}\left(C_{t^{\prime}} x\right)^{(k-2-j)}=\sum_{j=0}^{k-2} \sum_{i=j+2}^{k}\left(\begin{array}{l}
k-2-j \\
i-2-j
\end{array}\right) C_{t^{j}}^{(i-2-j)} x^{(k-i)} .
$$

Letting $\left(\begin{array}{l}i \\ j\end{array}\right)=0$ if $j<0$ or $i<j$, we may write

$$
\begin{aligned}
\sum_{j=0}^{k-2}\left(C_{t^{j}} x\right)^{(k-2-j)} & =\sum_{j=0}^{k-2} \sum_{i=2}^{k}\left(\begin{array}{c}
k-2-j \\
i-2-j
\end{array}\right) C_{t^{j}}^{(i-2-j)} x^{(k-i)} \\
& =\sum_{i=2}^{k} \sum_{j=0}^{k-2}\left(\begin{array}{c}
k-2-j \\
i-2-j
\end{array}\right) C_{t^{j}}^{(i-2-j)} x^{(k-i)} \\
& =\sum_{i=2}^{k} \sum_{j=2}^{k}\left(\begin{array}{l}
k-j \\
i-j
\end{array}\right) C_{t^{j-2}}^{(i-j)} x^{(k-i)} .
\end{aligned}
$$

This completes the proof of the relation (5.10). 
Let us now differentiate (5.7) $(n-1)$ times to obtain

$$
\begin{aligned}
x^{(n+1)}=(Q x)^{(n)}+(d / d t) & {\left[(G x)^{n-1}+(C x)^{(n-2)}+\left(C_{t} x\right)^{n-3}+\cdots\right.} \\
& \left.+C_{t^{n-2}} x+\int_{0}^{t}\left[\partial^{n-1} C(t, s) / \partial t^{n-1}\right] x(s) d s\right] .
\end{aligned}
$$

Let

$$
\begin{gathered}
\alpha_{k}=(G x)^{(k-1)}+\sum_{j=0}^{k-2}\left(C_{t^{j}} x\right)^{(k-2-j)}, \\
\beta_{k}=\sum_{i=1}^{k}\left(\begin{array}{c}
k \\
i
\end{array}\right) Q^{(i)} x^{(k-i)},
\end{gathered}
$$

and

$$
\gamma_{k}(i)=\left(\begin{array}{c}
k \\
i
\end{array}\right) Q^{(i)}-Q\left(\begin{array}{c}
k-1 \\
i-1
\end{array}\right) G^{(i-1)}-Q \sum_{j=2}^{i}\left(\begin{array}{c}
k-j \\
i-j
\end{array}\right) C_{t^{j-z}}^{(i-j)}
$$

for $k=1,2, \ldots, n$ and $i=1,2, \ldots, k$, assuming, of course, $\Sigma_{i}^{j} a_{i}=0$ if $j<i$. Using (5.10), we may write (5.12) as

$$
\alpha_{k}=\sum_{i=1}^{k}\left[\left(\begin{array}{c}
k-1 \\
i-1
\end{array}\right) G^{(i-1)}+\sum_{j=2}^{i}\left(\begin{array}{c}
k-j \\
i-j
\end{array}\right) C_{t^{j-2}}^{(i-j)}\right] x^{(k-i)}
$$

Also, (5.13)-(5.15) yield

$$
\beta_{k}-Q \alpha_{k}=\sum_{i=1}^{k} \gamma_{k}(i) x^{(k-1)}
$$

Thus, condition (a) reduces to

$$
\sum_{i=1}^{k}\left|\gamma_{k}(i)\right|+|Q| \int_{0}^{t}\left|\partial^{k-i} C(t, s) / \partial t^{k-i}\right| d s \leqslant R Q_{1},
$$

for $k=1,2, \ldots, n$. Also, equation (5.11) takes the form

$$
x^{(n+1)}=(Q x)^{(n)}+(d / d t)\left[\alpha_{n}+\int_{0}^{t}\left[\partial^{n-1} C(t, s) / \partial t^{n-1}\right] x(s) d s\right] .
$$

Proof OF THEOREM 17. We use induction on $n$. If $n=1$, conditions (a) and (b) of Theorem 17 reduce to (v) and (vi) of Theorem 16. Thus, Theorem 17 is true for $n=1$.

We now assume that Theorem 17 holds for $r=n-1, n \geqslant 2$, and show it holds for $r=n$.

Let $W=W\left(t, x(\cdot), x^{\prime}(\cdot), \ldots, x^{(n)}(\cdot)\right)$ and consider the functional

$$
W=\left(x^{(n)}-\left[\alpha_{n}+\int_{0}^{t}\left[\partial^{n-1} C(t, s) / \partial t^{n-1}\right] x(s) d s\right]\right)^{2} .
$$


Using (5.18), the derivative of $W$ with respect to $t$ along the solution $x(t)=x\left(t, t_{0}, \varphi\right)$ of $(5.1)$ is

$$
\begin{aligned}
W^{\prime}= & 2\left(x^{(n)}-\left[\alpha_{n}+\int_{0}^{t}\left[\partial^{n-1} C(t, s) / \partial t^{n-1}\right] x(s) d s\right]\right)(Q x)^{(n)} \\
= & 2\left(x^{(n)}-\left[\alpha_{n}+\int_{0}^{t}\left[\partial^{n-1} C(t, s) / \partial t^{n-1}\right] x(s) d s\right]\right)\left(Q x^{(n)}+\beta_{n}\right) \\
= & 2 Q\left[x^{(n)}\right]^{2}+2\left[\beta_{n}-Q \alpha_{n}-Q \int_{0}^{t}\left[\partial^{n-1} C(t, s) / \partial t^{n-1}\right] x(s) d s\right] x^{(n)} \\
& -2 \alpha_{n} \beta_{n}-2 \beta_{n} \int_{0}^{t}\left[\partial^{n-1} C(t, s) / \partial t^{n-1}\right] x(s) d s .
\end{aligned}
$$

By (5.16), we have

$$
\begin{aligned}
W^{\prime}= & 2 Q\left[x^{(n)}\right]^{2}+2 \sum_{i=1}^{n} \gamma_{n}(i) x^{(n-i)} x^{(n)}-2 Q \int_{0}^{t}\left[\partial^{n-1} C(t, s) / \partial t^{n-1}\right] x(s) x^{(n)} d s \\
& -2 \alpha_{n} \beta_{n}-2 \beta_{n} \int_{0}^{t}\left[\partial^{n-1} C(t, s) / \partial t^{n-1}\right] x(s) d s \\
\leqslant & 2 Q\left[x^{(n)}\right]^{2}+\sum_{i=1}^{n}\left|\gamma_{n}(i)\right|\left(\left[x^{(n-i)}\right]^{2}+\left[x^{(n)}\right]^{2}\right) \\
& +|Q| \int_{0}^{t}\left|\partial^{n-1} C(t, s) / \partial t^{n-1}\right|\left(x^{2}(s)+\left[x^{(n)}\right]^{2}\right) d s \\
& +2\left|\alpha_{n}\right|\left|\beta_{n}\right|+2\left|\beta_{n}\right| \int_{0}^{t}\left|\partial^{n-1} C(t, s) / \partial t^{n-1}\right||x(s)| d s .
\end{aligned}
$$

As $G=-\int_{t}^{\infty} C(u, t) d u$, we have

$$
G^{(k)}=\sum_{j=0}^{k-1} C_{s^{j}}^{(k-j-1)}-\int_{t}^{\infty}\left[\partial^{k} C(u, t) / \partial t^{k}\right] d u
$$

for $k=1,2, \ldots, n$.

Now, assume that (a) and (b) hold for $k=1,2, \ldots, n$. Then $C^{(k-1)}$ is bounded for $k=1,2, \ldots, n$ and hence $C_{t^{j}}^{(k-j-1)}$ and $C_{s^{j}}^{(k-j-1)}$ are bounded for $j=0,1,2, \ldots, k-1$. Thus, by (5.19) and (b), $G^{(k)}$ is bounded for $k=1,2, \ldots, n$. As (5.17) implies that $\gamma_{k}(i)$ is bounded for $k=1,2, \ldots, n$ and $i=1,2, \ldots, k$, then by $(5.14)$ we have $Q^{(i)}$ bounded for $i=1,2, \ldots, n$. Thus, there is a constant $L$ such that $\left|\alpha_{n}\right| \leqslant L \sum_{i=0}^{n-1}\left|x^{(i)}\right|$, $\left|\beta_{n}\right| \leqslant L \sum_{i=0}^{n-1}\left|x^{(i)}\right|$, and $\left|\gamma_{n}(i)\right| \leqslant L$ for $i=1,2, \ldots, n$. Hence,

$$
\begin{aligned}
2\left|\alpha_{n}\right|\left|\beta_{n}\right| & \leqslant 2 L^{2} \sum_{i=0}^{n-1} \sum_{j=0}^{n-1}\left|x^{(i)}\right|\left|x^{(j)}\right| \\
& \leqslant L^{2} \sum_{i=0}^{n-1} \sum_{j=0}^{n-1}\left(\left[x^{(i)}\right]^{2}+\left[x^{(j)}\right]^{2}\right) \leqslant L_{1} \sum_{i=0}^{n-1}\left[x^{(i)}\right]^{2}
\end{aligned}
$$


for some constant $L_{1}$. Thus,

$$
\begin{aligned}
W^{\prime} \leqslant & {\left[2 Q+\sum_{i=1}^{n}\left|\gamma_{n}(i)\right|+|Q| \int_{0}^{t}\left|\partial^{n-1} C(t, s) / \partial t^{n-1}\right| d s\right]\left[x^{(n)}\right]^{2} } \\
& +L \sum_{i=1}^{n}\left[x^{(n-i)}\right]^{2}+|Q| \int_{0}^{t}\left|\partial^{n-1} C(t, s) / \partial t^{n-1}\right| x^{2}(s) d s \\
& +L \sum_{i=0}^{n-1}\left[x^{(i)}\right]^{2}+L \sum_{i=0}^{n-1} \int_{0}^{t}\left|\partial^{n-1} C(t, s) / \partial t^{n-1}\right|\left(\left[x^{(i)}\right]^{2}+[x(s)]^{2}\right) d s
\end{aligned}
$$

By (b), $\int_{0}^{t}\left|\partial^{n-1} C(t, s) / \partial t^{n-1}\right| d s$ is bounded. Thus, using (5.17) and (i), we obtain

$$
\begin{aligned}
W^{\prime} \leqslant & {\left[-2 Q_{1}+R Q_{1}\right]\left[x^{(n)}\right]^{2}+L_{2} \sum_{i=0}^{n-1}\left[x^{(i)}\right]^{2} } \\
& +L_{3} \int_{0}^{t}\left|\partial^{n-1} C(t, s) / \partial t^{n-1}\right| x^{2}(s) d s
\end{aligned}
$$

for some constants $L_{2}$ and $L_{3}$. Now, the theorem is true for $r=n-1$. Thus $x^{(i)}(t)$ is in $L^{2}\left[t_{0}, \infty\right)$ for $i=0,1, \ldots, n-1$. Hence, we may define the functional $V=$ $V\left(t, x(\cdot), x^{\prime}(\cdot), \ldots, x^{(n)}(\cdot)\right)$ by

$$
\begin{aligned}
V= & W+K \sum_{i=0}^{n-1} \int_{t}^{\infty}\left[x^{(i)}(s)\right]^{2} d s \\
& +M \int_{0}^{t} \int_{t}^{\infty}\left|\partial^{n-1} C(u, s) / \partial u^{n-1}\right| d u x^{2}(s) d s \\
& +M \int_{t}^{\infty} \int_{s}^{\infty}\left|\partial^{n-1} C(u, s) / \partial u^{n-1}\right| d u x^{2}(s) d s
\end{aligned}
$$

and differentiate $V$ along the solution $x(t)=x\left(t, t_{0}, \varphi\right)$ of $(5.1)$ to obtain

$$
\begin{aligned}
V^{\prime}= & W^{\prime}-K \sum_{i=0}^{n-1}\left[x^{(i)}\right]^{2}+M \int_{t}^{\infty}\left|\partial^{n-1} C(u, t) / \partial u^{n-1}\right| d u x^{2} \\
& -M \int_{0}^{t}\left|\partial^{n-1} C(t, s) / \partial t^{n-1}\right| x^{2}(s) d s-M \int_{t}^{\infty}\left|\partial^{n-1} C(u, t) / \partial u^{n-1}\right| d u x^{2} .
\end{aligned}
$$

If we let $q=2 Q_{1}-R Q_{1}$ and make use of (5.20), we get

$$
\begin{aligned}
V^{\prime} \leqslant & -q\left[x^{(n)}\right]^{2}+\left(L_{2}-K\right) \sum_{i=0}^{n-1}\left[x^{(i)}\right]^{2} \\
& +\left(L_{3}-M\right) \int_{0}^{\infty}\left|\partial^{n-1} C(t, s) / \partial t^{n-1}\right| x^{2}(s) d s
\end{aligned}
$$

Choose $K$ and $M$ sufficiently large so that $V^{\prime} \leqslant-q\left[x^{(n)}\right]^{2}$ for all $t \geqslant t_{0}$. As $V \geqslant 0$, we conclude that $x^{(n)}(t)$ is in $L^{2}\left[t_{0}, \infty\right)$.

To show that $x^{(n)}(t) \rightarrow 0$ as $t \rightarrow \infty$, we differentiate (5.1) and (5.4) $k$ times to obtain

$$
x^{(k+1)}=(A x)^{(k)}+\sum_{j=0}^{k-1}\left(C_{t} j x\right)^{(k-1-j)}+\int_{0}^{t}\left[\partial^{k} C(t, s) / \partial t^{k}\right] x(s) d s
$$


and

$$
Q^{(k)}=A^{(k)}-G^{(k)}, \quad k=1,2, \ldots, n .
$$

As $Q^{(k)}$ and $G^{(k)}$ are bounded, we have $A^{(k)}$ bounded for $k=1,2, \ldots, n$.

Now, the theorem is true for $r=n-1$. Thus, $x^{(i)} t \rightarrow 0$ as $t \rightarrow \infty$ for $i=0,1, \ldots, n$ -1 . Hence, by (5.21) and (b), we have $x^{(n)}(t)$ bounded. Consequently, by (5.21), $x^{(n+1)}(t)$ is bounded and since $\left[x^{(n)}\right]^{2}$ is in $L^{1}\left[t_{0}, \infty\right]$, then $\left(\left[x^{(n)}\right]^{2}\right)^{\prime}=2 x^{(n)} x^{(n+1)}$ is bounded; thus $x^{(n)}(t) \rightarrow 0$ as $t \rightarrow \infty$. This completes the proof of Theorem 17 .

EXAMPLE 3. Consider the equation

$$
x^{\prime}=-\left[1+b(2 t+1)^{-2} / 2\right] x+b \int_{0}^{t}(t+s+1)^{-3} x(s) d s, \quad b>0
$$

where

$$
G(t, t)=-\int_{t}^{\infty} C(u, t) d u=-b(2 t+1)^{-2} / 2
$$

and $Q(t)=A(t)-G(t, t)=-1$. Thus, for any given positive integer $n$, we may choose $b$ sufficiently small so that all conditions of Theorem 17 are satisfied.

\section{REFERENCES}

1. E. A. Barbashin, The construction of Lyapunow functions, Differential Equations 4 (1968), 1097-1112 $=$ Differentsial'nye Uravneniya 4 (1968), 2127-2158. (Russian)

2. F. Brauer, Asymptotic stability of a class of integrodifferential equation, J. Differential Equations 28 (1978), 180-188.

3. T. A. Burton, Stability theory for Volterra equations, J. Differential Equations 32 (1979), 101-118.

4. R. D. Driver, Existence and stability of solutions of a delay-differential system, Arch. Rational Mech. Anal. 10 (1962), 401-426.

5. J. K. Hale, Ordinary differential equations, Wiley, New York, 1969.

6. E. Hewitt and K. Stromberg, Real and abstract analysis, Springer, New York, 1965.

7. G. S. Jordan, Asymptotic stability of a class of integrodifferential systems, J. Differential Equations 31 (1979), 359-365.

8. J. J. Levin, The asymptotic behavior of the solutions of a Volterra equation, Proc. Amer. Math. Soc. 14 (1963), 534-541.

9. R. K. Miller, Asymptotic stability properties of linear Volterra integrodifferential equations, J. Differential Equations 10 (1971), 485-506.

Department of Mathematics, Southern Illinois University, Carbondale, Illinois 62901

Department of Mathematics, Murray State University, Murray, Kentucky 42071 